\title{
Conception sécurisée des structures soumises aux valeurs extrêmes de processus stochastiques stationnaires
}

\author{
Bruno Colin ${ }^{\mathrm{a}}$ \\ Nexter Systems, Direction Technique, 11 allée des Marronniers, 78022 Versailles Cedex, France
}

Reçu le 10 janvier 2009, accepté le 3 mars 2009

\begin{abstract}
Résumé - Face à la nécessité d'accroître les performances de mobilité tout terrain de ses véhicules et à sa volonté d'y intégrer désormais des équipements « low cost» provenant du secteur civil, la Société Nexter s'est dotée de démarches de conception sécurisantes, permettant de faire face à ce type de compromis antinomique. Basée sur la capacité d'évaluer rapidement les valeurs des contraintes extrêmes produites sur les structures embarquées, dès le début du cycle de développement du produit, l'originalité de la démarche de conception proposée réside dans le fait qu'elle permet de synthétiser un grand nombre de processus d'excitation stochastique, décrits par le profil d'emploi opérationnel des clients, prendre en compte le caractère aléatoire des excitations concernées, en supposant de surcroît une indépendance statistique des situations stochastiques retenues, et de revenir à un chargement dynamique unique, reproduisant fidèlement les valeurs des contraintes extrêmes des processus concernés, avec un risque de dépassement maîtrisé puisque spécifié. Éprouvée dans le cadre du développement du VBCI (Véhicule Blindé de Combat d'Infanterie), cette démarche de conception dynamique a notamment mis en évidence le caractère peu sécurisant des démarches de conception forfaitaire à caractère statique déployées dans le passé.
\end{abstract}

Mots clés : Processus stochastique / dynamique des structures / contraintes extrêmes à risque de dépassement / SRX / processus de personnalisation / situations statistiquement indépendantes

\begin{abstract}
Design secure structures subjected to extreme values of stationary stochastic process. Faced with the need to increase the mobility requirement of his all-terrain vehicles and to integrate "low cost" equipment from the civil market, Nexter Systems has developed an approach to design safe, enabling to cope with this kind of antagonist compromise. Based on the ability to quickly calculate the extremes values of stress process which are produced on the structures, at the beginning of the development cycle of products, the originality of this approach design is that it allows to synthesize a large number of stochastic process, which are described by the operational profile life of customers, to take into account the random nature of excitations process and to suppose the statistical independence of the stochastic situations, to calculate a single dynamic loading, faithfully reproducing the extremes values of concerned processes, with a risk of exceeding controlled and specified. Tested in the VBCI development (Armoured Combat Vehicle), this dynamic approach has particularly highlighted the lack of safe which is associated to the static approach, deployed in the past.
\end{abstract}

Key words: Stochastic process / dynamic of the structures / extremes values with a risk of exceeding controlled / SRX / tailoring process / statistically independent process

\section{Introduction}

Les concepteurs de structures mécaniques sont souvent confrontés au fait d'optimiser très vite et sans risque leur concept d'architecture, en termes de comportement modal et de tenue mécanique.

Devant la complexité des profils d'emploi et la diversité des fonctions de service associées aux produits

\footnotetext{
a Auteur pour correspondance : b.colin@nexter-group.fr
}

d'Armements Terrestres, ce processus d'optimisation est bien souvent difficile à mener par les concepteurs, qui devant le nombre important de cas de chargement dynamique à réaliser se replient couramment sur des valeurs de chargement statique de nature forfaitaire et éprouvées par les développements antérieurs.

Si l'avantage de cette démarche de conception forfaitaire permet de dimensionner correctement les fixations des structures mécaniques, modélisées par EF, sans que 
l'on ait besoin de faire appel à des calculs plus sophistiqués, il ressort qu'elle ne permet pas d'optimiser efficacement le comportement dynamique de ces dernières. cela conduit en général à surdimensionner les structures sur leurs premiers modes de déformation, en ne sécurisant nullement le dimensionnement des modes élevés qui pour certaines situations d'utilisation sont fortement sollicités (cas des situations de roulage tout terrain à vitesses soutenues, et des situations de tir canon).

Ainsi, dans l'optique de pouvoir réellement optimiser le comportement dynamique des structures mécaniques, soumises à un grand nombre de processus stochastiques au cours de son profil de vie, il convenait de revenir inévitablement à des cas de chargement dynamique et non plus statique. Par ailleurs, l'objectif essentiel étant de disposer d'un processus de conception dynamique rapide et sécurisant, il convenait également de s'appuyer sur une démarche capable de synthétiser un grand nombre de Situations d'utilisation, au sens des contraintes extrêmes susceptibles d'être générées par ces dernières sur les structures à dimensionner.

De ces considérations techniques, le processus de conception mis en place par Nexter Systems pour dimensionner favorablement ses structures d'interface entre son véhicule porteur et les équipements étagères de ses fournisseurs s'appuie sur les grands principes suivants, à savoir :

- caractérisation en termes de contraintes extrêmes de chacune des situations d'utilisation, en gérant pour des raisons « sécuritaires » leur risque de dépassement que l'on s'impose a priori, en fonction de la criticité de l'équipement à installer sur la structure porteuse (notion de SRX [1]);

- regroupement et synthèse des situations du profil d'emploi, au sens des valeurs de contraintes extrêmes à risque de dépassement $\alpha$ imposé, en s'appuyant sur un processus de synthèse stochastique des situations considérées ;

- détermination d'une spécification de conception, sous forme d'une seule donnée d'excitation dynamique, couvrant avec «un bon niveau de représentativité » les valeurs de contraintes extrêmes susceptibles d'être générés au travers des modes de la structure mécanique à optimiser.

La méthodologie mise en place par Nexter Systems dans le processus de conception de ses structures d'interface est ici très largement empruntée « au processus de personnalisation des essais » [2], utilisé par les responsables de qualification des produits et que l'on peut adapter aux besoins des concepteurs en phase de développement.

L'originalité de la méthodologie présentée par Nexter Systems réside dans le fait quelle est basée sur un processus classique de calcul dynamique prenant en compte le caractère aléatoire des différentes excitations du profil d'emploi, et que l'on améliore par des techniques statistiques au titre de la synthèse des situations, que l'on suppose statistiquement indépendantes entre elles.

\section{Densité de probabilité des maxima locaux d'un processus aléatoire}

En restant dans le contexte des véhicules à roues (type VBCI $[3,4]$ ), on peut montrer que les processus d'excitation produits par ce type de véhicule, en situation de roulage tout terrain et/ou tout chemin, sont de nature aléatoire et stationnaire à vitesse constante. Ces derniers sont par ailleurs de forts niveaux, compte tenu de l'accroissement des performances de mobilité dévolues à ce type de plate-forme de mobilité, qui de par son architecture de train de roulement $(8 \times 8$ à suspension oléopneumatique [5], propulsée par un moteur diesel de 550 ch couplé à une boîte de vitesses automatiques) lui permet d'évoluer à des vitesses importantes et soutenues sur des profils tout terrain à fortes rugosités $(23 \mathrm{~cm}$ de rugosité efficace à plus de $45 \mathrm{~km} \cdot \mathrm{h}^{-1}$ ).

De ces considérations techniques, il ressort que les processus d'excitation aléatoire de roulage tout terrain constituent donc des cas de chargement dynamique dimensionnants pour les structures embarquées sur véhicules terrestres à forte mobilité. Pour caractériser l'effet dimensionnant de ces processus d'excitation aléatoire, il convient dans un premier temps d'en évaluer les valeurs extrêmes sur la durée d'excitation finie, imposée par le profil d'utilisation du client.

\subsection{Loi générale ou loi complète}

En se plaçant dans le cadre des processus aléatoires gaussiens $Z(t, \xi)$ à large bande, il est possible de définir la formulation analytique de la loi de densité de probabilité de ses maxima locaux, en se basant sur la théorie de Rice [6] qui dans les années 1944 fut le premier auteur à démontrer ce résultat fondamental. Ces travaux ont été consolidés par ceux de Cartwright et Longuet-Higgins en 1956 [7], qui constituent aujourd'hui les deux références bibliographiques majeures dans l'étude des maxima des processus stochastiques de nature gaussienne. Ainsi en considérant un processus aléatoire gaussien à large bande $Z(t, \xi)$, de valeur moyenne nulle et de valeur efficace $\sigma_{z}$, Rice dans les années 44 montre que la loi de densité de probabilité des maxima locaux $Z_{\max }(\xi)=Z\left(t_{i}, \xi\right)$ peut s'écrire sous la forme suivante, à savoir :

$$
\begin{aligned}
f_{Z_{\max }}(z)= & \left.\frac{g_{\eta_{\max }}(u)}{\sigma_{z}} \text { où } u=\frac{z}{\sigma_{z}} \text { et } z \in\right]-\infty,+\infty[ \\
\text { et } g_{\eta_{\max }}(u)= & \frac{\sqrt{1-r^{2}}}{\sqrt{2 \pi}} \exp \left[-\frac{u^{2}}{2\left(1-r^{2}\right)}\right]+\frac{r}{2} u \\
& \times \exp \left(-\frac{u^{2}}{2}\right)\left[1+\operatorname{erf}\left(\frac{r u}{\sqrt{2\left(1-r^{2}\right)}}\right)\right]
\end{aligned}
$$

avec :

$\eta_{\max }(\xi)=\frac{Z_{\max }(\xi)}{\sigma_{z}}:$ maxima local réduit du processus aléatoire $Z(t, \xi)$,

$Z_{\max }(\xi)=Z\left(t_{i}, \xi\right)$ : maxima local du processus aléatoire $Z(t, \xi)$, 
$z$ : amplitude des maxima locaux du processus aléatoire $Z(t, \xi)$,

$r$ : facteur d'irrégularité associé au processus aléatoire $Z(t, \xi)$.

L'amplitude des maxima réduits du processus aléatoire $Z(t, \xi)$ est donc une valeur sans dimension et a pour expression :

$$
u=\frac{z}{\sigma_{z}}
$$

avec :

$\sigma_{z}$ : valeur efficace du processus aléatoire $Z(t, \xi)$.

Par ailleurs, la fonction erf(.) qui intervient dans l'expression (1) précédente correspond à la fonction erreur [8]. Cette dernière est une expression impaire, qui tend vers l'unité lorsque son argument tend vers l'infini. Son expression analytique est alors la suivante :

$$
\operatorname{erf}(x)=\frac{2}{\sqrt{\pi}} \int_{\lambda=0}^{x} \exp \left(-\lambda^{2}\right) \mathrm{d} \lambda
$$

Comme le montre la figure 1 qui suit, les maxima locaux $Z\left(t_{i}, \xi\right)$ de tels processus stochastiques sont alors caractérisés par la variable aléatoire $Z_{\max }(\xi)$ dont la densité de probabilité est définie par la relation (1). Cette dernière dépend du facteur d'irrégularité $r$ du processus aléatoire qui est une valeur comprise entre 0 et 1 , selon que le processus aléatoire étudié est à bande large ou à bande étroite. L'expression analytique de ce facteur d'irrégularité est donc :

$$
r=\frac{E\left[\overline{N_{0}^{+}}(\xi)\right]}{E\left[\overline{N_{\max }}(\xi)\right]}
$$

avec :

$E\left[\overline{N_{0}^{+}}(\xi)\right]$ : taux moyen de franchissement par zéro avec pente positive du processus aléatoire $Z(t, \xi)$. Cette expression est connue sous le nom de «formule de Rice » et est communément appelée « fréquence centrale et/ou fréquence moyenne $»$ du processus aléatoire considéré,

$E\left[\overline{N_{\max }}(\xi)\right]$ : taux moyen des maxima locaux du processus aléatoire $Z(t, \xi)$.

Les processus aléatoires gaussiens $Z(t, \xi)$ sont des processus stochastiques particuliers puisque l'on peut montrer qu'ils sont complètement définis par leur densité spectrale de puissance $D S P_{Z Z}(f)$, définie en terme de fréquence positive. Notamment cette DSP fréquentielle unilatérale permet de définir les expressions analytiques de $E\left[\overline{N_{0}^{+}}(\xi)\right]$ et de $E\left[\overline{N_{\max }}(\xi)\right]$ comme le montrent respectivement les relations (5) et (6). En effet en s'appuyant sur les moments spectraux de cette DSP, on peut écrire :

$$
E\left[\overline{N_{0}^{+}}(\xi)\right]=\frac{1}{2 \pi}\left(\frac{m_{2}}{m_{0}}\right)^{\frac{1}{2}}
$$

et,

$$
E\left[\overline{N_{\max }}(\xi)\right]=\frac{1}{2 \pi}\left(\frac{m_{4}}{m_{2}}\right)^{\frac{1}{2}}
$$

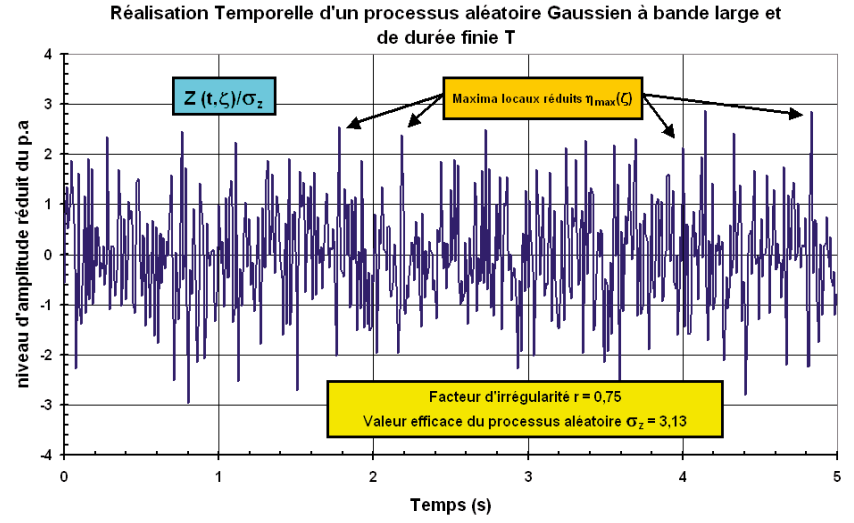

Fig. 1. Réalisation temporelle d'un processus aléatoire gaussien.

avec :

$m_{i}: i^{\text {ème }}$ moment spectral du processus aléatoire $Z(t, \xi)$.

Les moments spectraux de la DSP fréquentielle unilatérale sont alors définis par la relation fondamentale [8] suivante, à savoir :

$$
m_{i}=\int_{f=0}^{+\infty}(2 \pi f)^{i} D S P_{Z Z}(f) \mathrm{d} f
$$

De ce fait l'expression simplifiée du facteur d'irrégularité peut être établie pour les processus gaussiens, en injectant les relations (6) et (7) dans l'expression générale (4) et on obtient :

$$
r=\frac{m_{2}}{\sqrt{m_{0} m_{4}}}
$$

Ainsi les expressions précédentes montrent clairement que la d.d.p des maxima d'un processus aléatoire gaussien stationnaire et de largeur de bande quelconque peut être caractérisée uniquement en connaissant sa DSP fréquentielle unilatérale. En effet, on vient de voir que cette dernière permet alors d'estimer :

- le facteur d'irrégularité $r$ du p.a en s'appuyant sur (8);

- puis sa valeur efficace $\sigma_{z}=\sqrt{m_{0}}$ en s'appuyant $\operatorname{sur}(7)$;

- et sa d.d.p des maxima locaux $f_{Z_{\max }}(z)$ en s'appuyant $\operatorname{sur}(1)$.

Ainsi conformément à la réalisation du processus aléatoire gaussien $Z(t, \xi)$ de la figure 1 , il est possible de tracer la d.d.p de ses maxima locaux réduits, qui est une courbe à support positif et négatif, dans la mesure où ce processus admet des maxima à valeurs négatives (puisque ce dernier est à bande large, $r=0,75)$. En ce qui concerne la loi de d.d.p des maxima locaux réduits, il est également important de noter que cette courbe est de nature adimensionnelle et caractérise donc de ce fait complètement la statistique de ses maxima locaux. On a donc schématiquement la figure 2. 


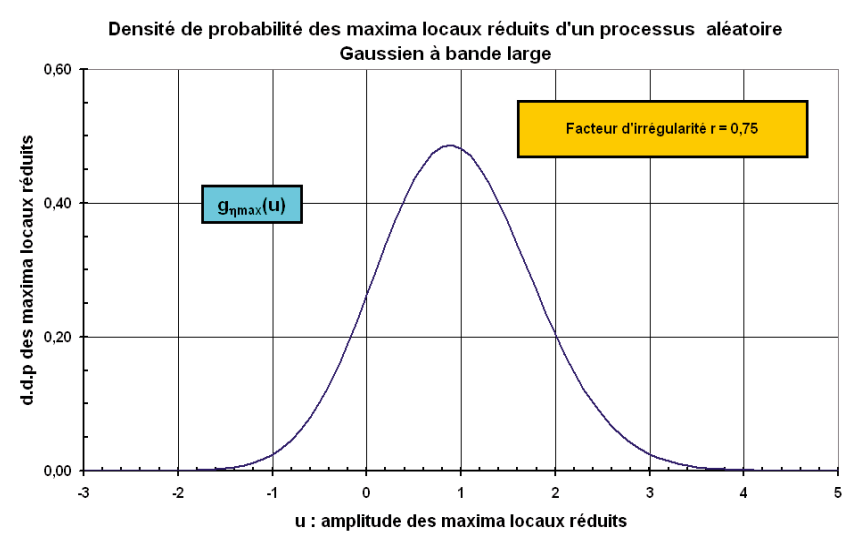

Fig. 2. Densité de probabilité des maxima locaux réduits d'un processus aléatoire gaussien.

\subsection{Cas d'un processus aléatoire à bande étroite}

Un processus à bande étroite est caractérisé par un facteur d'irrégularité unitaire, dans la mesure où un extrema (maxima ou minima) est forcément suivi d'un passage par zéro. De ce fait en posant $(r=1)$ dans la relation (1) précédente, on peut obtenir l'expression de la loi de densité de probabilité des maxima réduits du processus aléatoire à bande étroite et on obtient :

$$
\begin{aligned}
f_{Z_{\max }}(z) & =\frac{g_{\eta_{\max }}(u)}{\sigma_{z}} \quad \text { où } \quad u=\frac{z}{\sigma_{z}} \\
\text { et } \quad g_{\eta_{\max }}(u) & =u \exp \left(-\frac{u^{2}}{2}\right) \quad \text { avec } \quad u \geq 0
\end{aligned}
$$

Soit,

$$
f_{Z_{\max }}(z)=\frac{z}{\sigma_{z}^{2}} \exp \left(-\frac{z^{2}}{2 \sigma_{z}^{2}}\right) \quad \text { avec } \quad z \geq 0
$$

En s'appuyant sur l'expression (10) précédente, on constate que la loi de d.d.p des maxima locaux $f_{Z_{\max }}(z)$ d'un p.a gaussien à bande étroite est décrite par une loi de Rayleigh dont la forme adimensionnelle $g_{\eta_{\max }}(u)$ est présentée à la figure 3 .

\subsection{Cas d'un processus aléatoire à bande large}

Un processus à bande large est caractérisé par un facteur d'irrégularité nulle, dans la mesure où un grand nombre d'extrema (maxima ou minima) est forcément compris entre deux passages par zéro successifs. De ce fait en posant $(r=0)$ dans la relation (1) précédente, on peut obtenir l'expression de la loi de densité de probabilité des

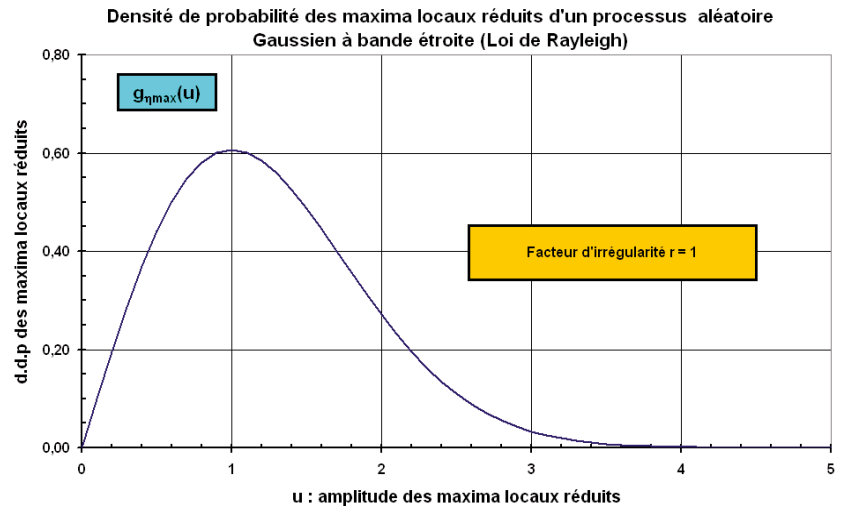

Fig. 3. Densité de probabilité des maxima locaux réduits d'un processus aléatoire gaussien à bande étroite $(r=1)$.

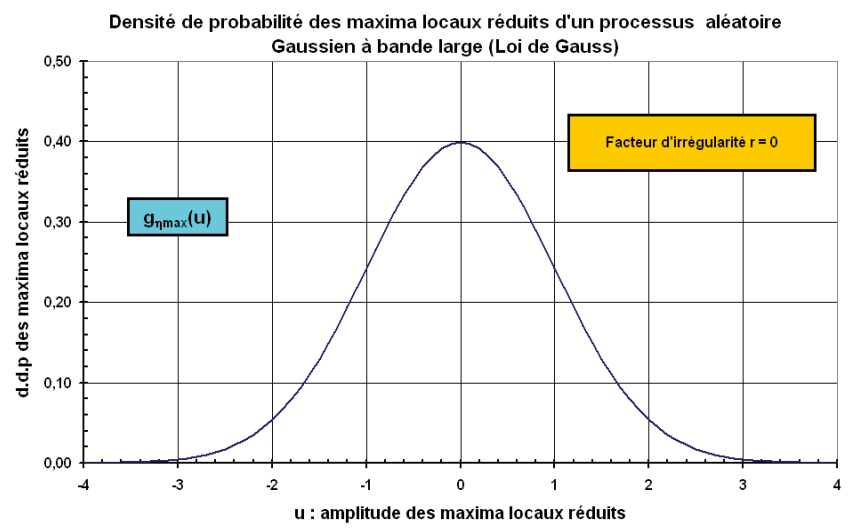

Fig. 4. Densité de probabilité des maxima locaux réduits d'un processus aléatoire gaussien à bande large $(r=0)$.

maxima réduits du processus aléatoire à bande large et on obtient :

$$
f_{Z_{\max }}(z)=\frac{g_{\eta_{\max }}(u)}{\sigma_{z}} \quad \text { où } \quad u=\frac{z}{\sigma_{z}}
$$

et $g_{\eta_{\max }}(u)=\frac{1}{\sqrt{2 \pi}} \exp \left(-\frac{u^{2}}{2}\right)$ avec $\left.u \in\right]-\infty,+\infty[$

Soit,

$$
\left.f_{Z_{\max }}(z)=\frac{1}{\sqrt{2 \pi} \sigma_{z}} \exp \left(-\frac{z^{2}}{2 \sigma_{z}^{2}}\right) \text { avec } z \in\right]-\infty,+\infty[
$$

En s'appuyant sur l'expression (12) précédente, on constate que la loi de d.d.p des maxima locaux $f_{Z_{\max }}(z)$ d'un p.a gaussien à bande large est décrite par une loi de Gauss dont la forme adimensionnelle $g_{\eta_{\max }}(u)$ est présentée à la figure 4 .

\section{Fonction de répartition des maxima locaux d'un processus aléatoire}

De part sa définition, la fonction de répartition $F_{Z_{\max }}\left(z_{0}\right)$ des maxima locaux d'un p.a, dont sa d.d.p des 


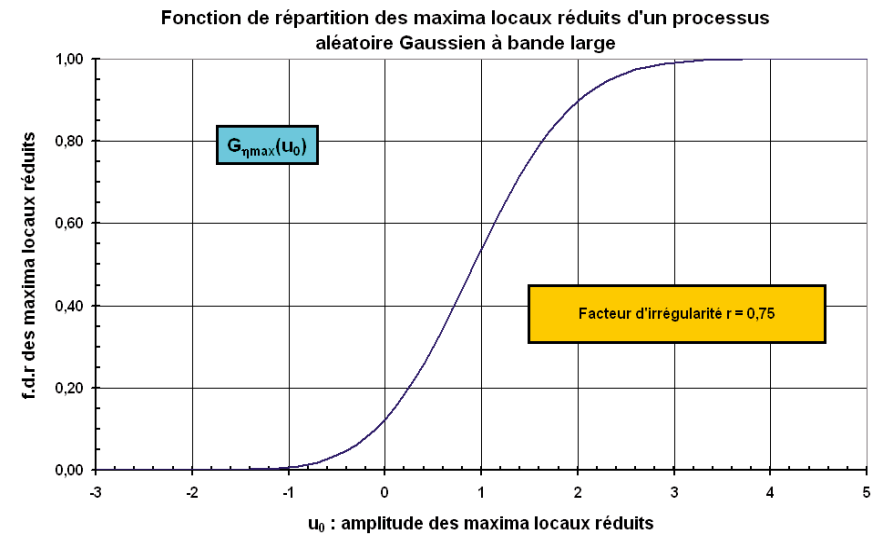

Fig. 5. Fonction de répartition des maxima locaux réduits d'un processus aléatoire gaussien.

maxima locaux $f_{Z_{\max }}(z)$ est connue, peut s'écrire de la façon suivante :

$$
F_{Z_{\max }}\left(z_{0}\right)=\operatorname{Prob}\left[Z_{\max }(\xi) \leq z_{0}\right]=\int_{-\infty}^{z_{0}} f_{Z_{\max }}(z) \mathrm{d} z
$$

En retenant cette fois la variable aléatoire adimensionnelle associée aux maxima locaux du p.a, on peut écrire :

$$
\begin{aligned}
G_{\eta_{\max }}\left(u_{0}\right) & =\operatorname{Prob}\left[\eta_{\max }(\xi) \leq u_{0}\right]=\int_{-\infty}^{u_{0}} g_{\eta_{\max }}(u) \mathrm{d} u \\
& =F_{Z_{\max }}\left(z_{0}\right) \quad \text { avec } \quad u_{0}=\frac{z_{0}}{\sigma_{z}}
\end{aligned}
$$

Ainsi en intégrant l'expression (1) de $-\infty$ à $u_{0}$, on peut obtenir l'expression générale de la loi de répartition des maxima locaux réduits d'un p.a gaussien et stationnaire, à savoir :

$$
\begin{aligned}
G_{\eta_{\max }}\left(u_{0}\right)= & F_{Z_{\max }}\left(z_{0}\right)=\frac{1}{2}\left\{1+\operatorname{erf}\left(\frac{u_{0}}{\sqrt{2\left(1-r^{2}\right)}}\right)\right. \\
& \left.-r \exp \left(-\frac{u_{0}^{2}}{2}\right)\left[1+\operatorname{erf}\left(\frac{r u_{0}}{\sqrt{2\left(1-r^{2}\right)}}\right)\right]\right\}
\end{aligned}
$$

avec $u_{0}=\frac{z_{0}}{\sigma_{z}}$ et $\left.z_{0} \in\right]-\infty,+\infty[$

Pour le p.a gaussien $Z(t, \xi)$ de la figure 1, il est alors possible de tracer la fonction de répartition (f.d.r) de ses maxima locaux réduits en s'appuyant sur la relation (15) précédente, ce qui conduit à tracer la figure 5 .

\subsection{Cas d'un processus aléatoire à bande étroite}

En posant $(r=1)$ dans la relation (15) précédente, on peut obtenir l'expression de la fonction de répartition des maxima réduits du processus aléatoire à bande étroite et on obtient :

$$
G_{\eta_{\max }}\left(u_{0}\right)=1-\exp \left(-\frac{u_{0}^{2}}{2}\right) \quad \text { avec } \quad u_{0} \geq 0
$$

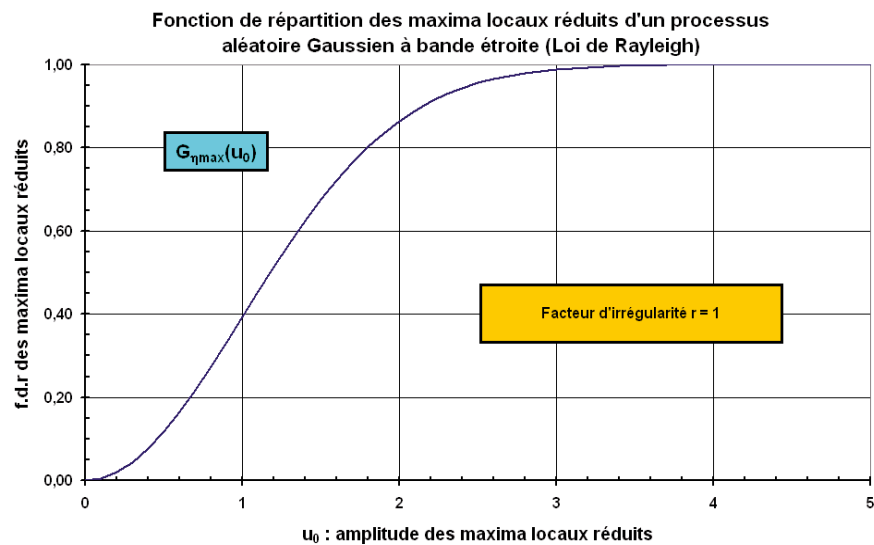

Fig. 6. Fonction de répartition des maxima locaux réduits d'un processus aléatoire gaussien à bande étroite $(r=1)$.

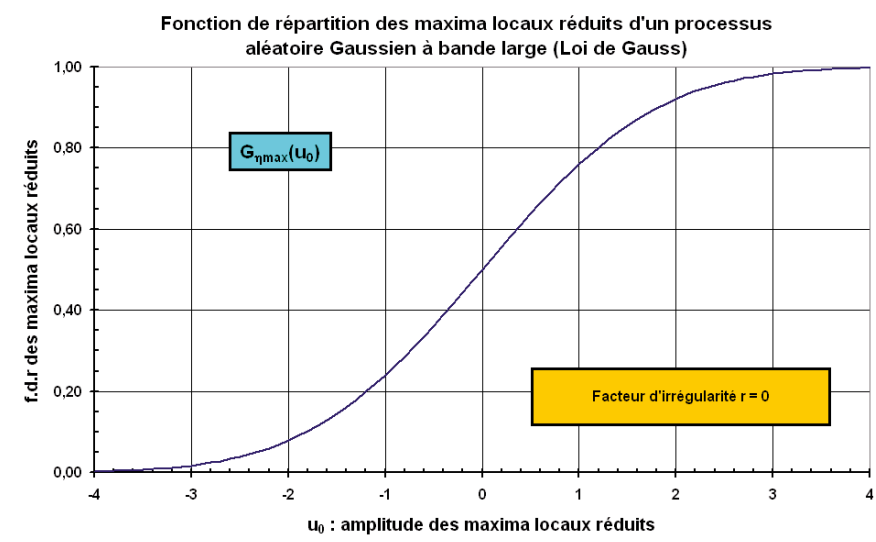

Fig. 7. Fonction de répartition des maxima locaux réduits d'un processus aléatoire gaussien à bande large $(r=0)$.

En s'appuyant sur l'expression (16) précédente, on constate que la fonction de répartition (f.d.r) des maxima locaux réduits $G_{\eta_{\max }}\left(u_{0}\right)$ d'un p.a gaussien à bande étroite est exprimée à partir d'une loi exponentielle décroissante dont la forme est décrite à la figure 6 .

\subsection{Cas d'un processus aléatoire à bande large}

En posant $(r=0)$ dans la relation (15) précédente, on peut obtenir l'expression de la fonction de répartition des maxima réduits du processus aléatoire à bande large et on obtient :

$$
\left.G_{\eta_{\max }}\left(u_{0}\right)=\frac{1}{2}\left[1+\operatorname{erf}\left(\frac{u_{0}}{\sqrt{2}}\right)\right] \quad \text { avec } \quad u_{0} \in\right]-\infty,+\infty[
$$

En s'appuyant sur l'expression (17) précédente, on constate que la fonction de répartition (f.d.r) des maxima locaux réduits $G_{\eta_{\max }}\left(u_{0}\right)$ d'un p.a gaussien à bande étroite est exprimée à partir de la fonction erreur dont l'expression intégrale est définie par (3). Sa représentation graphique est alors décrite à la figure 7 . 


\section{Densité de probabilité du plus grand maxima local d'un processus aléatoire}

Conformément à l'objectif recherché, il convient désormais de définir la loi de d.d.p des valeurs extrêmes d'un p.a gaussien stationnaire de durée $T$ finie. Pour ce faire, on va s'intéresser à la variable aléatoire (v.a) $Y_{n}(\xi)$ correspondant à la valeur maximale de $n$ variables aléatoires indépendantes $\left\{Z_{\max }, i(\xi) ; i=1\right.$ à $\left.n\right\}$, où chaque variable aléatoire $Z_{\max }, i(\xi)$ est calée sur la loi des maxima locaux du p.a $Z(t, \xi)$ considéré. En admettant que ces v.a sont indépendantes entre elles quelle que soit la largeur de bande du p.a considéré, on peut écrire d'emblée :

$$
\begin{aligned}
& F_{Y_{n}}(y)=\left[F_{Z_{\max }}(y)\right]^{n} \\
& \text { où } F_{Z_{\max }}(y) \text { est définie par }(15)
\end{aligned}
$$

avec pour le cas des p.a stationnaires de durée finie $T$, une valeur de $n$ calée sur le nombre moyen de maxima locaux du processus aléatoire $Z(t, \xi)$ qui s'écrit alors de la façon suivante :

$$
n=E\left[\overline{N_{\max }}(\xi)\right] T
$$

$E\left[\overline{N_{\max }}(\xi)\right]$ : taux moyen des maxima locaux du processus aléatoire $Z(t, \xi)$,

$T$ : durée de sollicitation du processus aléatoire $Z(t, \xi)$.

Connaissant la f.d.r de la v.a $Y_{n}(\xi)$, il est désormais possible d'en déduire sa loi de d.d.p, puisque l'on a par définition :

$$
f_{Y_{n}}(y)=\frac{\mathrm{d}\left[F_{Y_{n}}(y)\right]}{\mathrm{d} y}
$$

Ainsi en injectant (15) dans (18), puis (18) dans (20), on peut écrire :

$$
f_{Y_{n}}(y)=n\left[F_{Z_{\max }}(y)\right]^{n-1} f_{Z_{\max }}(y)
$$

où $F_{Z_{\max }}(y)$ est définie par (15)

et $f_{Z_{\max }}(y)$ est définie par (1)

Comme pour les maxima locaux, on va là encore raisonner en variable réduite et pour ce faire, on va définir une v.a $\gamma_{n}(\xi)$ qui sera associée au plus grand maxima réduit du processus aléatoire gaussien $Z(t, \xi)$, à savoir :

$$
\gamma_{n}(\xi)=\frac{Y_{n}(\xi)}{\sigma_{z}}
$$

En s'appuyant sur l'expression (22) et sur les définitions des lois de d.d.p et de f.d.r des v.a $Y_{n}(\xi)$ et $\gamma_{n}(\xi)$, on montre alors que :

- la f.d.r de la v.a $\gamma_{n}(\xi)$ a pour expression :

$$
G_{\gamma_{n}}\left(\nu_{0}\right)=F_{Y_{n}}\left(y_{0}\right) \quad \text { avec } \quad \nu_{0}=\frac{y_{0}}{\sigma_{z}}
$$

où $F_{Y_{n}}\left(y_{0}\right)$ est définie par (18)

- la d.d.p de la v.a $\gamma_{n}(\xi)$ a pour expression :

$$
g_{\gamma_{n}}\left(\nu_{0}\right)=\sigma_{z} f_{Y_{n}}\left(y_{0}\right) \quad \text { avec } \quad \nu_{0}=\frac{y_{0}}{\sigma_{z}}
$$

où $f_{Y_{n}}\left(y_{0}\right)$ est définie par (21)

\subsection{Cas d'un processus aléatoire à bande étroite}

En posant $(r=1)$ dans les relations (23) et (24) précédentes, il est possible de définir les lois statistiques (d.d.p et f.d.r) du plus grand maxima réduit d'un processus aléatoire gaussien, à bande étroite, stationnaire et de durée finie $T$. On obtient donc pour la fonction de répartition (f.d.r) :

$$
G_{\gamma_{n}}\left(\nu_{0}\right)=\left[1-\exp \left(-\frac{\nu_{0}^{2}}{2}\right)\right]^{n} \text { avec } n=E\left[\overline{N_{\max }}(\xi)\right] T
$$

Et pour la loi de densité de probabilité (d.d.p), on obtient :

$$
g_{\gamma_{n}}\left(\nu_{0}\right)=n \nu_{0}\left[1-\exp \left(-\frac{\nu_{0}^{2}}{2}\right)\right]^{n-1} \exp \left(-\frac{\nu_{0}^{2}}{2}\right)
$$

avec $n=E\left[\overline{N_{\max }}(\xi)\right] T$

\subsection{Cas d'un processus aléatoire à bande large}

De même, en posant $(r=0)$ dans les relations (23) et (24) précédentes, il est possible de définir les lois statistiques (d.d.p et f.d.r) du plus grand maxima réduit d'un processus aléatoire gaussien, à bande large, stationnaire et de durée finie $T$. On obtient donc pour la fonction de répartition (f.d.r) :

$$
G_{\gamma_{n}}\left(\nu_{0}\right)=\frac{\left[1+\operatorname{erf}\left(\frac{\nu_{0}}{\sqrt{2}}\right)\right]^{n}}{2^{n}} \operatorname{avec} n=E\left[\overline{N_{\max }}(\xi)\right] T
$$

Et pour la loi de densité de probabilité (d.d.p), on obtient :

$$
g_{\gamma_{n}}\left(\nu_{0}\right)=\frac{n}{\sqrt{2 \pi}} \frac{\left[1+\operatorname{erf}\left(\frac{\nu_{0}}{\sqrt{2}}\right)\right]^{n-1}}{2^{n-1}} \exp \left(-\frac{\nu_{0}^{2}}{2}\right)
$$

avec $n=E\left[\overline{N_{\max }}(\xi)\right] T$

\section{Valeur du plus grand maxima local d'un processus aléatoire, à risque de dépassement donné}

Pour sécuriser le dimensionnement des structures mécaniques soumises à des processus aléatoires gaussiens large bande, il convient désormais de s'intéresser aux valeurs extrêmes générées par ce type de p.a, en en recherchant la valeur du plus grand maxima local dépassée avec un risque $\alpha$ maîtrisé et spécifié. Ce concept est également utilisé dans le domaine des spécifications en environnement mécanique pour comparer favorablement les phénomènes déterministes (chocs et vibrations sinusoïdales) avec les phénomènes à caractère stochastique (vibrations aléatoires), en termes de contraintes extrêmes [1]. 
Valeurs du plus grand maxima réduit du p.a à bande large, associé à un risque de dépassement de $99 \%$

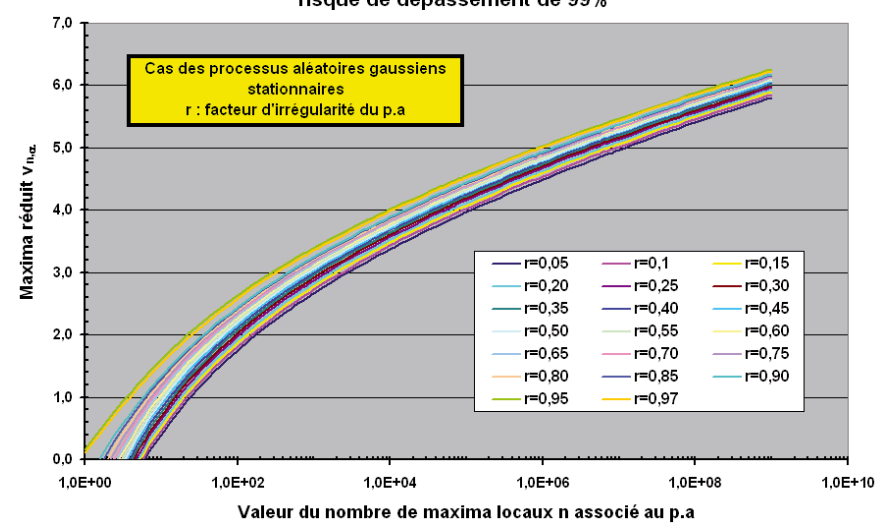

Fig. 8. Valeurs du plus grand maxima réduit $\nu_{n, \alpha}$, associées à un risque de dépassement de $99 \%$ (Figure disponible en couleurs sur www.mecanique-industries.org).

Par définition, cette valeur du plus grand maxima, à risque de dépassement $\alpha$ donné, est dénommée $y_{n, \alpha}$ et est obtenue en résolvant la relation (29) qui suit :

$$
F_{Y_{n}}\left(y=y_{n, \alpha}\right)=1-\alpha
$$

où $F_{Y_{n}}(y)$ est définie par (18)

De ce fait l'expression analytique de $y_{n, \alpha}$ s'écrit de la façon suivante, sachant qu'elle se résout uniquement par voie numérique dans le cas général des p.a gaussiens de largeur de bande quelconque. En effet, on se doit de résoudre l'expression suivante pour trouver la valeur recherchée $y_{n, \alpha}$ :

$$
y_{n, \alpha}=F_{Y_{n}}^{-1}(1-\alpha)
$$

avec :

$\alpha$ : risque de dépassement imposé (on retiendra communément une valeur de $1 \%$ pour sécuriser la conception. Dans certains cas une valeur de $0,1 \%$ sera considérée si le risque de rupture peut entraîner des dommages au niveau des personnels).

Compte tenu des niveaux de risque de dépassement faibles adoptés, les algorithmes de résolution numérique doivent travailler avec une précision importante et de l'ordre de $1 E-20$ pour estimer les valeurs de $y_{n, \alpha}$, lorsque $n$ est de fort niveau. Ce travail de résolution a été effectué pour deux valeurs de risque, à savoir $\alpha=99 \%$ (Fig. 8) et $\alpha=1 \%$ (Fig. 9), en considérant une plage de valeurs de $n$ comprise entre 1 et $1 E+09$.

Par ailleurs, dans l'optique de disposer d'abaques universels et transposables quels que soient le niveau énergétique et la durée du p.a considéré, ces abaques sont tracés en exprimant la valeur du plus grand maxima réduit $\nu_{n, \alpha}$ en fonction de la valeur $n$ du nombre des maxima locaux du processus considéré.

La valeur du plus grand maxima réduit du p.a s'exprime donc de la façon suivante :

$$
\nu_{n, \alpha}=\frac{y_{n, \alpha}}{\sigma_{z}}
$$

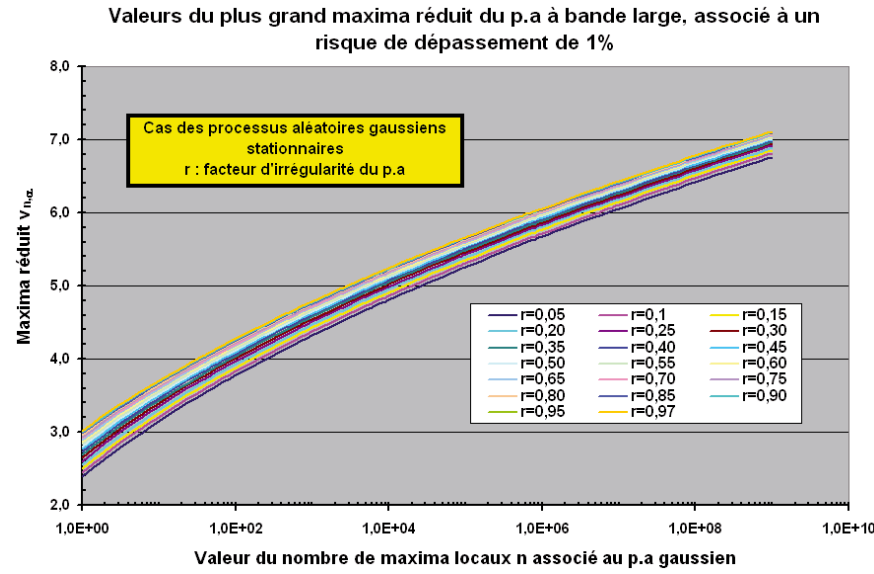

Fig. 9. Valeurs du plus grand maxima réduit $\nu_{n, \alpha}$, associées à un risque de dépassement de $1 \%$ (Figure disponible en couleurs sur www.mecanique-industries.org).

Ainsi pour un risque de dépassement donné, cet abaque adimensionnel (et donc utilisable quels que soient le niveau et la durée du p.a considéré), met en évidence clairement les deux points importants suivants :

- pour une forme spectrale donnée du p.a (et donc pour une valeur de facteur d'irrégularité donnée et un niveau efficace donné), la valeur du maxima réduit à risque de dépassement donné croît avec la durée de sollicitation du p.a. Ceci montre donc que plus le processus stochastique sera de durée importante et plus la sévérité du dimensionnement devra être élevée, tout paramètre égal par ailleurs ;

- pour une même durée et un même niveau efficace de sollicitation, la valeur du maxima réduit à risque de dépassement augmente avec la diminution de largeur de bande du processus stochastique considéré. Ceci montre donc qu'il est toujours préférable de prédimensionner les structures en utilisant la théorie de Rayleigh (bande étroite) plutôt que celle de Gauss (bande large).

En comparant les deux abaques précédents des figures 8 et 9 , on montre également clairement que plus le risque de dépassement spécifié est faible et plus la valeur du maxima réduit est élevée, ce qui constitue somme toute, un résultat logique. Par ailleurs, ces abaques mettent en évidence le caractère peu sécurisant des démarches de dimensionnement statique, basées sur des critères forfaitaires consistant à dimensionner les structures mécaniques à 3 fois les valeurs efficaces des processus de contrainte.

Pour s'en convaincre, on voit qu'au sens d'un risque de dépassement de $1 \%$, il faudrait retenir une valeur de contrainte maximale située entre 5 et 6 fois sa valeur efficace pour dimensionner favorablement une structure, dont le premier mode serait situé à $30 \mathrm{~Hz}$ et pour lequel la durée de sollicitation serait de $1 \mathrm{~h}$. De plus comme les structures mécaniques ne possèdent généralement pas qu'un seul mode prépondérant, il devient également souhaitable de disposer de démarches 


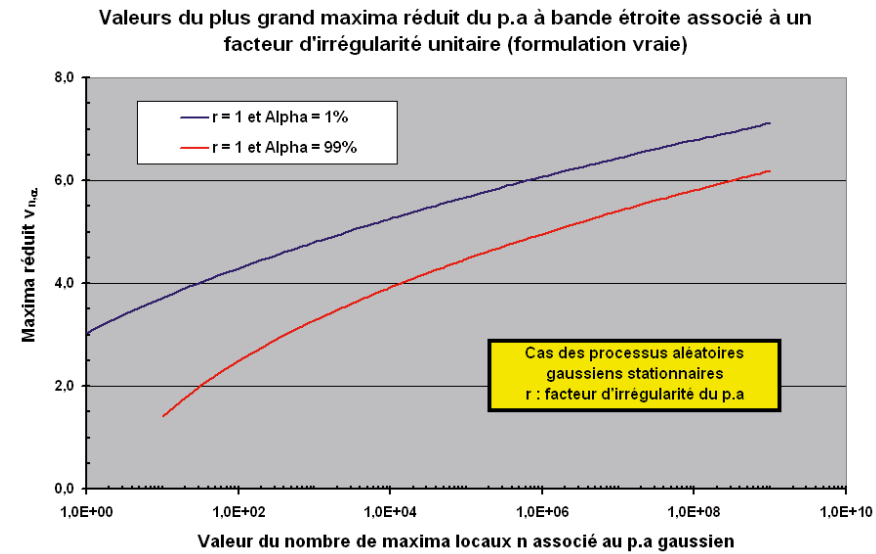

Fig. 10. Valeurs du plus grand maxima réduit $\nu_{n, \alpha}$ d'un p.a gaussien à bande étroite, associées aux risques de dépassement de $1 \%$ et $99 \%$ (Figure disponible en couleurs sur www. mecanique-industries.org).

de conception sécurisantes prenant en compte le comportement dynamique de ces dernières, et éviter ainsi les risques de sous dimensionnement de certains modes élevés.

\subsection{Cas d'un processus aléatoire à bande étroite}

En injectant la relation (25) dans la relation (29), il est possible d'écrire d'emblée :

$$
\left[1-\exp \left(-\frac{\nu_{\alpha}^{2}}{2}\right)\right]^{n}=1-\alpha
$$

D'où : $\nu_{\alpha} s=\sqrt{-2 \ln \left[1-(1-\alpha)^{\frac{1}{n}}\right]}$

Par ailleurs pour une valeur de risque $\alpha$ petite (cas de $1 \%$ ), on peut montrer en s'appuyant sur un développement de Mac Laurin que :

$$
(1-\alpha)^{\frac{1}{n}} \underset{\alpha \text { petit }}{\longrightarrow} 1-\frac{\alpha}{n}
$$

d'où en injectant (33) dans (32) :

$$
\nu_{\alpha} \underset{\alpha \text { petit }}{\longrightarrow} \sqrt{2 \ln \left[\frac{n}{\alpha}\right]}
$$

La figure 10 montre combien le choix de la valeur de risque est déterminant pour estimer les valeurs de contraintes maximales d'un p.a gaussien et ceci quelle que soit la valeur de $n$ retenue.

\subsection{Cas d'un processus aléatoire à bande large}

En injectant la relation (27) dans la relation (29), il est possible d'écrire d'emblée :

$$
\left[1+\operatorname{erf}\left(\frac{\nu_{\alpha}}{\sqrt{2}}\right)\right]^{n}=2^{n}(1-\alpha)
$$

Valeurs du plus grand maxima réduit du p.a à bande large, associé à un facteur d'irrégularité nul (formulation simplifiée)

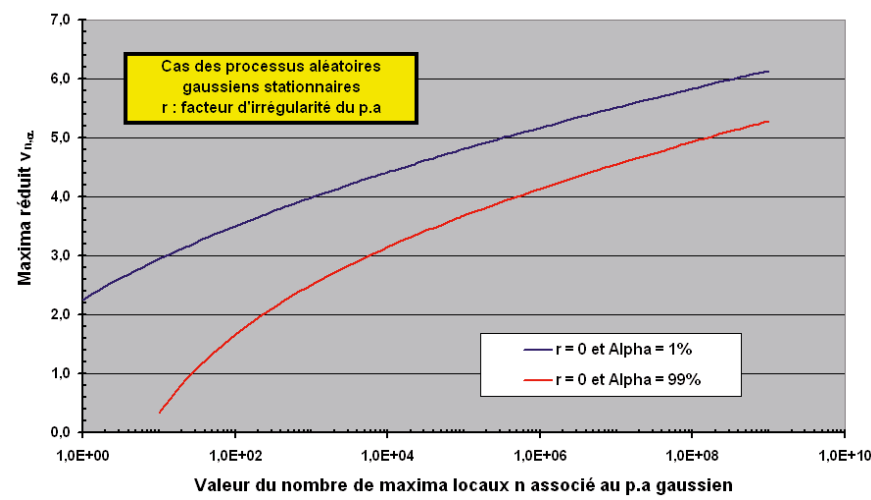

Fig. 11. Valeurs du plus grand maxima réduit $\nu_{n, \alpha}$ d'un p.a gaussien à bande large, associées aux risques de dépassement de $1 \%$ et $99 \%$ (Figure disponible en couleurs sur www. mecanique-industries.org).

D'où : $\nu_{\alpha}=\sqrt{2} \operatorname{erf}^{-1}\left(2(1-\alpha)^{\frac{1}{n}}-1\right)$

À ce stade, il est possible de proposer une formulation approchée du plus grand maxima réduit $\nu_{\alpha}$ en se basant sur la formulation approchée de la fonction erreur qui suit [11] et dont le niveau d'erreur est inférieur à quelques pour mille, quelle que soit la valeur de son argument :

$$
\operatorname{erf}(x) \approx \sqrt{1-\exp \left(-\frac{4 x^{2}}{\pi}\right)}
$$

Ainsi en injectant (36) dans (35), on obtient la valeur de $\nu_{\alpha}$ pour un processus à bande large, à savoir :

$$
\nu_{\alpha} \approx \sqrt{-\frac{\pi}{2} \ln \left[1-\left(2(1-\alpha)^{\frac{1}{n}}-1\right)^{2}\right]}
$$

Dans le cas où le risque de dépassement est petit et sur la base de l'approximation (33), il est également possible d'écrire :

$$
\nu_{\alpha} \underset{\alpha \text { petit }}{\longrightarrow} \sqrt{-\frac{\pi}{2} \ln \left[\frac{4 \alpha}{n}\left(1-\frac{\alpha}{n}\right)\right]}
$$

Les écarts constatés entre la formulation vraie fournie par la relation (35) et la formulation approchée fournie par (37) sont de l'ordre de 7 à $10 \%$ sur la plage des valeurs de $n$ retenue (à savoir $[1,1 \mathrm{E}+09]$ ) et pour le domaine des valeurs de risque $\alpha$ considéré (à savoir $[1 \%$, $99 \%]$ ).

\section{Expression asymptotique de la fonction de répartition du plus grand maxima local d'un processus aléatoire}

La loi de d.d.p des maxima locaux (1) étant asymptotiquement décrite par une décroissance exponentielle, on peut montrer que la f.d.r du plus grand maxima local 
$Y_{n}(\xi)$ d'un p.a gaussien tend vers une loi de Gumbel [11] dont l'expression est :

$$
F_{Y_{n}}(y) \underset{n \text { grand }}{\longrightarrow} \exp \left[-\exp \left(-\alpha_{n}\left(y-y_{n}\right)\right)\right]
$$

avec :

$y_{n}$ : valeur caractéristique maximale d'ordre $n$ du processus aléatoire $Z(t, \xi)$,

$\alpha_{n}$ : valeur de l'intensité extrême maximale d'ordre $n$ du processus aléatoire $Z(t, \xi)$.

De par leurs définitions, on peut donc écrire d'emblée :

$$
y_{n}=F_{Z_{\max }}^{-1}\left(1-\frac{1}{n}\right)
$$

où $F_{Z_{\max }}(y)$ est définie par (15)

$$
\alpha_{n}=n f_{Z_{\max }}\left(y_{n}\right)
$$

où $f_{Z_{\max }}(y)$ est définie par (1)

Ainsi connaissant la d.d.p et la f.d.r des maxima locaux du p.a gaussien, il est possible de trouver la valeur de $y_{n}$ en s'appuyant sur (40) et donc la valeur de $\alpha_{n}$ en injectant (40) dans (41). Néanmoins comme on se situe dans le cas des valeurs importantes de $n$, il est possible de proposer des expressions analytiques des valeurs de $y_{n}$ et de $\alpha_{n}$, en s'appuyant sur l'expression asymptotique de la fonction erreur [7] qui s'écrit :

$$
\operatorname{erf}(x) \underset{x \text { grand }}{\longrightarrow} 1-\frac{1}{\sqrt{\pi} x \exp \left(x^{2}\right)}
$$

\subsection{Expression asymptotique de $y_{n}$}

En injectant (42) dans l'expression (15) et en faisant tendre $n$ vers l'infini, on peut montrer que :

$$
\begin{aligned}
& G_{\eta_{\max }}\left(u_{n}\right)=F_{Z_{\max }}\left(z_{n}\right) \underset{n \text { grand }}{\longrightarrow} 1-r \exp \left(-\frac{u_{n}^{2}}{2}\right) \\
& \text { où } \quad u_{n}=\frac{z_{n}}{\sigma_{z}}
\end{aligned}
$$

d'où l'expression asymptotique de $y_{n}$, en injectant (43) dans (40) et en s'appuyant sur les relations (4) et (19) :

$$
y_{n} \underset{n \text { grand }}{\longrightarrow} \sigma_{z} \sqrt{2 \ln (r n)}=\sigma_{z} \sqrt{2 \ln \left(E\left[\overline{N_{0}^{+}}(\xi)\right]\right)}
$$

Nota: On constate que l'expression asymptotique de $y_{n}$ est exploitable quelle que soit la largeur de bande du p.a gaussien concerné, excepté dans le cas où $r$ est nulle (p.a gaussien à bande large).

En raisonnant en variable adimensionnelle, on montre donc que la valeur caractéristique maximale d'ordre $n$ réduite du p.a $Z(t, \xi)$ a pour expression :

$$
\nu_{n}=\frac{y_{n}}{\sigma_{z}} \underset{n \text { grand }}{\longrightarrow} \sqrt{2 \ln (r n)}
$$

En posant $r=1$ dans l'expression (45), on obtient l'expression de $y_{n}$ pour le cas des processus aléatoires gaussiens à bande étroite, qui est un résultat démontré dans [1] en injectant directement (16) dans (40). On obtient donc pour $y_{n}$ et par conséquent pour $\nu_{n}$ les résultats classiques suivants :

$$
\begin{gathered}
y_{n}=\frac{\nu_{n}}{\sigma_{z}} \quad \text { avec } \quad \nu_{n}=\sqrt{2 \ln (n)} \forall n \quad \text { pour } \quad r=1 \\
\text { avec } n=E\left[\overline{N_{\max }}(\xi)\right] T=E\left[\overline{N_{0}^{+}}(\xi)\right] T
\end{gathered}
$$

Comme indiqué précédemment les formulations (44) et (45) ne peuvent pas s'appliquer au cas des processus aléatoires à bande large $(r=0)$, il convient donc désormais d'injecter l'expression (17) dans (40) pour obtenir l'expression de $y_{n}$ utilisable pour le cas des p.a gaussiens à bande large $(r=0)$. Ainsi en raisonnant en variable adimensionnelle, on obtient donc l'expression de $\nu_{n}$ suivante, à savoir :

$$
\nu_{n}=\sigma_{z} y_{n}=\sqrt{2} \operatorname{erf}^{-1}\left(1-\frac{2}{n}\right) \forall n \quad \text { pour } \quad r=0
$$

avec $n=E\left[\overline{N_{\max }}(\xi)\right] T$

En utilisant de plus l'expression simplifiée (36) de la fonction erreur erf $(x)$, on obtient l'expression simplifiée suivante de $\nu_{n}$ pour le cas des processus aléatoires gaussiens stationnaires à bande large $(r=0)$ :

$$
\begin{aligned}
\nu_{n} & \approx \sqrt{-\frac{\pi}{2} \ln \left[\frac{4}{n}\left(1-\frac{1}{n}\right)\right]} \forall n \text { pour } r=0 \\
\text { avec } n & =E\left[\overline{N_{\max }}(\xi)\right] T
\end{aligned}
$$

\subsection{Expression asymptotique de $\alpha_{n}$}

En injectant (42) dans l'expression (1) et en faisant tendre $n$ vers l'infini, on peut montrer que :

$$
g_{\eta_{\max }}\left(u_{n}\right)=\sigma_{z} f_{Z_{\max }}\left(z_{n}\right) \underset{n \text { grand }}{\longrightarrow} r u_{n} \exp \left(-\frac{u_{n}^{2}}{2}\right)
$$

où $u_{n}=\frac{z_{n}}{\sigma_{z}}$

d'où l'expression asymptotique de $\alpha_{n}$, en injectant (44) et (49) dans (41) :

$$
\alpha_{n} \underset{n \text { grand }}{\longrightarrow} \frac{y_{n}}{\sigma_{z}^{2}} \quad \text { avec } \quad y_{n}=\sigma_{z} \sqrt{2 \ln (r n)}
$$

Nota : On constate que la valeur $\alpha_{n}$ n'est pas une valeur adimensionnelle puisqu'elle est homogène à l'inverse de l'amplitude du p.a $Z(t, \xi)$ considéré. La valeur adimensionnelle $\beta_{n}$ à prendre en compte est donc :

$$
\beta_{n}=\sigma_{z} \alpha_{n}=\nu_{n}=\sqrt{2 \ln (r n)}
$$

puisque $\quad \alpha_{n}=\frac{y_{n}}{\sigma_{z}^{2}}$

En posant $r=1$ dans l'expression (50), on obtient l'expression de $\alpha_{n}$ pour le cas des processus aléatoires gaussiens à bande étroite, qui est un résultat démontré dans [1] en injectant directement (9) dans (41). On obtient donc 
pour $\alpha_{n}$ et par conséquent pour $\beta_{n}$ les résultats classiques suivants :

$$
\begin{aligned}
& \beta_{n}=\sigma_{z} \alpha_{n}=\nu_{n} \text { avec } \nu_{n}=\sqrt{2 \ln (n)} \\
& \forall n \quad \text { pour } r=1
\end{aligned}
$$

avec $n=E\left[\overline{N_{\max }}(\xi)\right] T=E\left[\overline{N_{0}^{+}}(\xi)\right] T$

Les formulations (50) et (51) ne peuvent pas s'appliquer au cas des processus aléatoires à bande large $(r=0)$, il convient donc désormais d'injecter l'expression (12) dans (41) pour obtenir l'expression de $\alpha_{n}$ utilisable pour le cas des p.a gaussiens à bande large $(r=0)$. Ainsi en raisonnant en variable adimensionnelle, on obtient donc l'expression de $\beta_{n}$ suivante, à savoir :

$$
\begin{aligned}
& \beta_{n}=\sigma_{z} \alpha_{n}=\frac{n}{\sqrt{2 \pi}} \exp \left[-\left[\operatorname{erf}^{-1}\left(1-\frac{2}{n}\right)\right]^{2}\right] \\
& \forall n \text { pour } r=0
\end{aligned}
$$

avec $n=E\left[\overline{N_{\max }}(\xi)\right] T$

En utilisant de plus l'expression simplifiée (36) de la fonction erreur erf $(x)$, on obtient l'expression simplifiée suivante de $\beta_{n}$ pour le cas des processus aléatoires gaussiens stationnaires à bande large $(r=0)$ :

$$
\begin{aligned}
& \beta_{n} \approx \frac{n}{\sqrt{2 \pi}} \exp \left[\frac{\pi}{4} \ln \left[\frac{4}{n}\left(1-\frac{1}{n}\right)\right]\right] \forall n \quad \text { pour } \quad r=0 \\
& \text { avec } n=E\left[\overline{N_{\max }}(\xi)\right] T
\end{aligned}
$$

\subsection{Expression asymptotique de $F_{Y_{n}}(y)$}

Des considérations précédentes, on montre donc que l'expression asymptotique de la f.d.r du plus grand maxima local du p.a gaussien peut s'écrire de la façon suivante dans le cas où les valeurs de $n$ sont élevées :

$$
F_{Y_{n}}(y) \underset{n \text { grand }}{\longrightarrow} \exp \left[-\exp \left(-\alpha_{n} y+\beta_{n} \nu_{n}\right)\right]
$$

avec $\nu_{n}=\sqrt{2 \ln (r n)}$ et $\beta_{n}=\sigma_{z} \alpha_{n}=\nu_{n}$

\section{Expression asymptotique de la densité de probabilité du plus grand maxima local d'un processus aléatoire}

Connaissant désormais l'expression asymptotique de la f.d.r du plus grand maxima local du p.a gaussien, il est possible d'en évaluer sa d.d.p, en appliquant par définition [10] la relation suivante :

$$
f_{Y_{n}}(y) \underset{n \text { grand }}{\longrightarrow} \frac{\mathrm{d} F_{Y_{n}}(y)}{\mathrm{d} y}
$$

avec $F_{Y_{n}}(y)$ définie par $(47)$
On obtient donc :

$$
\begin{aligned}
& f_{Y_{n}}(y) \underset{n \text { grand }}{\longrightarrow} \alpha_{n} \exp \left[-\alpha_{n} y+\beta_{n} \nu_{n}\right] \\
& \quad \times \exp \left[-\exp \left[-\alpha_{n} y+\beta_{n} \nu_{n}\right]\right] \\
& \text { avec } \nu_{n}=\sqrt{2 \ln (r n)}=\frac{y_{n}}{\sigma_{z}} \quad \text { et } \beta_{n}=\sigma_{z} \alpha_{n}=\nu_{n}
\end{aligned}
$$

\subsection{Moyenne et variance asymptotique de $Y_{n}(\xi)$}

À partir de cette d.d.p asymptotique, associée à la v.a $Y_{n}(\xi)$ correspondant au plus grand maxima local du processus aléatoire gaussien $Z(t, \xi)$, il est possible de définir les expressions analytiques de sa moyenne et de sa variance. Pour ce faire, on s'appuie sur les définitions de base de ces deux quantités statistiques [10].

Par définition la valeur moyenne de la v.a $Y_{n}(\xi)$ a pour expression :

$$
E\left[Y_{n}(\xi)\right]=\int_{y=-\infty}^{y=+\infty} y f_{Y_{n}}(y) \mathrm{d} y
$$

et sa variance a pour expression :

$$
\begin{aligned}
\operatorname{Var}\left[Y_{n}(\xi)\right] & =E\left[Y_{n}^{2}(\xi)\right]-\left(E\left[Y_{n}(\xi)\right]\right)^{2} \\
E\left[Y_{n}^{2}(\xi)\right] & =\int_{y=-\infty}^{y=+\infty} y^{2} f_{Y_{n}}(y) \mathrm{d} y
\end{aligned}
$$

En injectant l'expression asymptotique (57) dans les relations (58) et (59), on montre que l'on peut obtenir :

$$
E\left[Y_{n}(\xi)\right] \underset{n \text { grand }}{\longrightarrow} y_{n}+\frac{\varepsilon}{\alpha_{n}}
$$

avec $\varepsilon=$ Cste d'Euler $=0,5772156 \ldots$

Cette expression est en accord avec celle fournie par Cartwright et Longuet-Higgins dans les années 1956 [7].

Et en ce qui concerne la variance de la v.a $Y_{n}(\xi)$, on peut montrer également que :

$$
\operatorname{Var}\left[Y_{n}(\xi)\right] \underset{n \text { grand }}{\longrightarrow} \frac{\pi^{2}}{6 \alpha_{n}^{2}}
$$

\subsection{Facteur de pic du processus aléatoire $Z(t, \xi)$}

La moyenne asymptotique de $Y_{n}(\xi)$, ramenée à la valeur efficace $\sigma_{z}$ du p.a $Z(t, \xi)$ est présentée par un grand nombre d'auteurs comme étant le facteur de pic $\delta_{z}$ du p.a gaussien considéré. Ce coefficient $\delta_{z}$ est souvent utilisé par les concepteurs pour dimensionner leurs structures soumises à des excitations aléatoires de nature gaussienne.

Son expression est :

$$
\begin{aligned}
& \delta_{z}=\frac{E\left[Y_{n}(\xi)\right]}{\sigma_{z}} \underset{n \text { grand }}{\longrightarrow} \nu_{n}+\frac{\varepsilon}{\beta_{n}} \text { avec } \nu_{n}=\beta_{n}=\sqrt{2 \ln (r n)} \\
& \text { et } \varepsilon=\text { Cste d'Euler }=0,57721566490 \ldots
\end{aligned}
$$




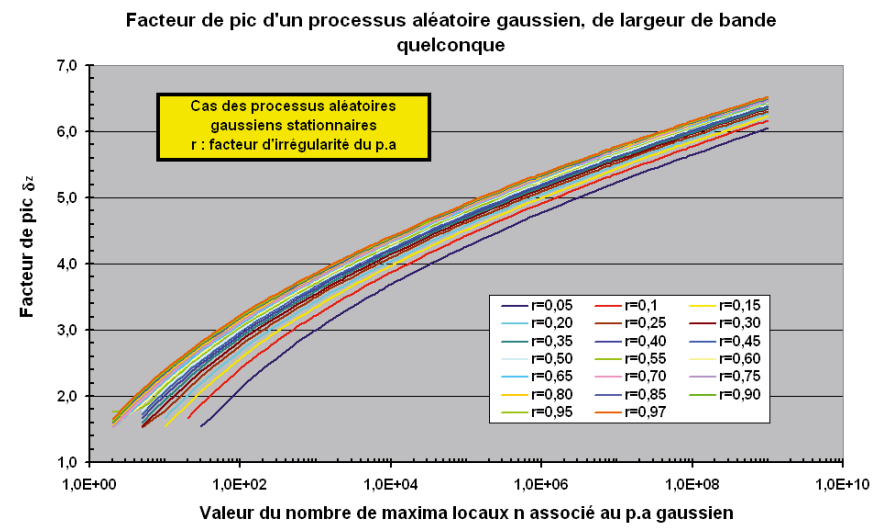

Fig. 12. Facteur de pic $\delta_{z}$ d'un p.a gaussien à bande large (Figure disponible en couleurs sur www.mecanique-industries. org).

En posant $r=1$ dans l'expression (62), on obtient l'expression du facteur de pic $\delta_{z}$ pour le cas des processus aléatoires gaussiens à bande étroite, qui est un résultat classiquement utilisé par les concepteurs. On obtient donc l'expression suivante :

$$
\delta_{z} \underset{n \text { grand }}{\longrightarrow} \sqrt{2 \ln (n)}+\frac{\varepsilon}{\sqrt{2 \ln (n)}} \text { pour } r=1
$$

avec $n=E\left[\overline{N_{\max }}(\xi)\right] T=E\left[\overline{N_{0}^{+}}(\xi)\right] T$

et $\varepsilon=$ Cste d'Euler $=0,57721566490 \ldots$

La formulation (62) ne peut pas s'appliquer brutalement au cas des processus aléatoires à bande large $(r=0)$. Pour ce faire, il convient d'utiliser, pour $\nu_{n}$ et $\beta_{n}$, les expressions obtenues respectivement aux relations (48) et (54). Ainsi on peut écrire :

$\delta_{z} \underset{n \text { grand }}{\longrightarrow} \nu_{n}+\frac{\varepsilon}{\beta_{n}}$ avec $\varepsilon=$ Cste d'Euler $=0,57721566490 \ldots$

$$
\text { et } \begin{aligned}
\nu_{n} \approx \sqrt{-\frac{\pi}{2} \ln \left[\frac{4}{n}\left(1-\frac{1}{n}\right)\right]} \\
\beta_{n} \approx \frac{n}{\sqrt{2 \pi}} \exp \left[\frac{\pi}{4} \ln \left[\frac{4}{\pi}\left(1-\frac{1}{n}\right)\right]\right]
\end{aligned}
$$

L'expression du facteur de pic pour un p.a gaussien large bande est ici valable pour des valeurs de $n$ élevées. Cette nouvelle formulation basée sur l'expression générale de $E\left[Y_{n}(\xi)\right]$, définie par (60), peut être comparée à l'expression asymptotique fournie dés les années 1928 par Fisher et Tippett [13], à savoir :

$$
\delta_{z}=\frac{E\left[Y_{n}(\xi)\right]}{\sigma_{z}} \underset{n \text { grand }}{\longrightarrow} \sqrt{2 \ln \left(\frac{n}{\sqrt{2 \pi}}\right)} \text { pour } \quad r=0
$$

On constate que pour les valeurs élevées de $n$, le modèle asymptotique de Fisher et Tippet se situe au dessus de la courbe $(r=0,05)$, alors que logiquement le modèle pour

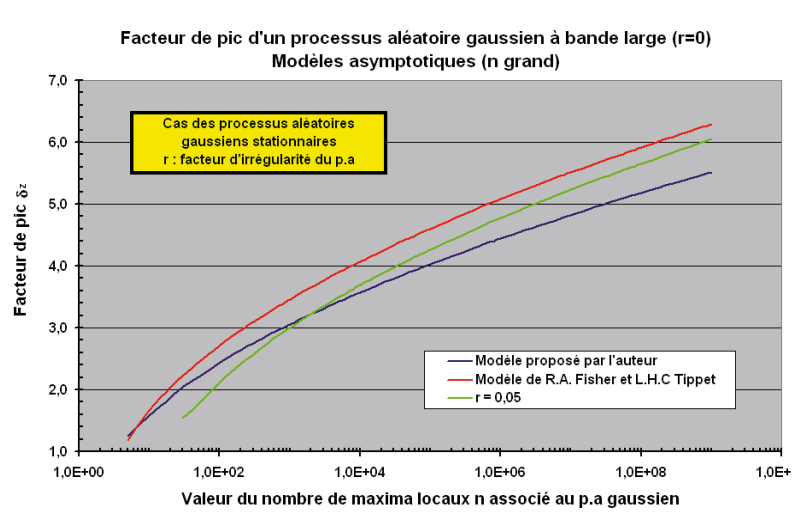

Fig. 13. Facteur de pic $\delta_{z}$ d'un p.a gaussien à bande large $(r=0)$. Cas du modèle de Fisher et Tippet, comparé au modèle de l'auteur (Figure disponible en couleurs sur www. mecanique-industries.org).

$(r=0)$ devrait se situer en dessous de cette courbe. De ce fait le modèle de l'auteur semble fournir des tendances plus réalistes puisque ce dernier se situe en dessous de la courbe $(r=0,05)$.

\section{Valeur asymptotique du plus grand maxima local d'un processus aléatoire, à risque de dépassement donné}

Connaissant désormais les expressions asymptotiques de la d.d.p et de la f.d.r du plus grand maxima local d'un processus aléatoire gaussien, il est alors possible de déterminer l'expression asymptotique du plus grand maxima local d'un p.a, à risque de dépassement $\alpha$ donné. Ainsi en injectant l'expression asymptotique (55) dans la relation (29) du plus grand maxima local à risque de dépassement $\alpha$, on peut alors montrer que la valeur asymptotique de $y_{n, \alpha}$ a pour expression :

$$
\begin{array}{r}
y_{n, \alpha} \underset{n \text { grand }}{\longrightarrow} y_{n}-\frac{\ln [-\ln (1-\alpha)]}{\alpha_{n}} \\
\text { avec } y_{n}=\sigma_{z} \sqrt{2 \ln (r n)} \text { et } \alpha_{n}=\frac{y_{n}}{\sigma_{z}^{2}}
\end{array}
$$

La valeur asymptotique et réduite du plus grand maxima local, à risque de dépassement, $\nu_{\alpha, n}$ est donc obtenue en injectant l'expression (66) dans (31), ce qui conduit à écrire :

$$
\nu_{n, \alpha} \underset{n \text { grand }}{\longrightarrow} \nu_{n}-\frac{\ln [-\ln (1-\alpha)]}{\beta_{n}}
$$

avec $\nu_{n}=\sqrt{2 \ln (r n)}$ et $\beta_{n}=\sigma_{z} \alpha_{n}=\frac{y_{n}}{\sigma_{z}}=\nu_{n}$

À ce stade, il devient désormais possible de comparer les vraies valeurs de $\nu_{\alpha, n}$, issues du modèle décrit par (29) et (31) avec les valeurs asymptotiques de $\nu_{\alpha, n}$, issues du modèle décrit par (67). Par ailleurs compte tenu de l'objectif recherché, cette comparaison est effectuée uniquement pour le cas du risque de dépassement faible $\alpha=1 \%$. 
Tableau 1. Comparaison des valeurs $\nu_{\alpha, n}$ entre le modèle «vrai » et le modèle « asymptotique » pour $\alpha=1 \%$.

\begin{tabular}{|c|c|c|c|c|c|c|c|c|c|}
\hline $\begin{array}{l}\text { Facteur d'irrégularité } \\
\text { (r sans dimension) }\end{array}$ & 0,1 & 0,2 & 0,3 & 0,4 & 0,5 & 0,6 & 0,7 & 0,8 & 0,9 \\
\hline $\begin{array}{l}n: \text { valeur du nombre de maxima } \\
\quad \text { locaux du p.a de durée } T\end{array}$ & \multicolumn{9}{|c|}{$\begin{array}{c}\text { Valeur asymptotique du plus grand maxima local réduit d'un p.a gaussien (Ecart en } \% \\
\text { entre la Formulation «vraie» et la Formulation «asymptotique» pour un risque de } \\
\text { dépassement } \alpha=1 \% \text { ) }\end{array}$} \\
\hline 1 & 1 & 1 & 1 & 1 & 1 & 1 & 1 & 1 & 1 \\
\hline 2 & 1 & 1 & 1 & 1 & 1 & 165,75 & 104,52 & 79,40 & 65,33 \\
\hline 5 & 1 & 1 & 88,70 & 55,99 & 43,00 & 35,81 & 31,19 & 27,95 & 25,54 \\
\hline 10 & 1 & 53,78 & 35,19 & 27,81 & 23,64 & 20,90 & 18,96 & 17,51 & 16,37 \\
\hline 20 & 49,30 & 26,34 & 20,48 & 17,41 & 15,43 & 14,04 & 13,00 & 12,19 & 11,55 \\
\hline 30 & 30,37 & 19,56 & 16,02 & 13,96 & 12,56 & 11,55 & 10,78 & 10,18 & 9,69 \\
\hline 40 & 23,16 & 16,30 & 13,73 & 12,13 & 11,01 & 10,18 & 9,55 & 9,05 & 8,65 \\
\hline 50 & 19,26 & 14,34 & 12,29 & 10,96 & 10,00 & 9,29 & 8,74 & 8,31 & 7,95 \\
\hline 100 & 11,92 & 10,13 & 9,07 & 8,26 & 7,65 & 7,17 & 6,80 & 6,51 & 6,26 \\
\hline 200 & 8,07 & 7,58 & 7,00 & 6,48 & 6,05 & 5,72 & 5,46 & 5,25 & 5,07 \\
\hline 300 & 6,60 & 6,53 & 6,12 & 5,70 & 5,35 & 5,07 & 4,86 & 4,68 & 4,53 \\
\hline 400 & 5,78 & 5,91 & 5,59 & 5,23 & 4,92 & 4,68 & 4,49 & 4,33 & 4,20 \\
\hline 500 & 5,24 & 5,49 & 5,22 & 4,90 & 4,62 & 4,40 & 4,23 & 4,08 & 3,96 \\
\hline 600 & 4,85 & 5,18 & 4,95 & 4,66 & 4,40 & 4,20 & 4,03 & 3,90 & 3,79 \\
\hline 700 & 4,56 & 4,94 & 4,74 & 4,47 & 4,23 & 4,03 & 3,88 & 3,75 & 3,65 \\
\hline 800 & 4,32 & 4,74 & 4,57 & 4,31 & 4,08 & 3,90 & 3,75 & 3,63 & 3,53 \\
\hline 900 & 4,12 & 4,58 & 4,43 & 4,18 & 3,96 & 3,79 & 3,65 & 3,53 & 3,44 \\
\hline 1000 & 3,96 & 4,44 & 4,30 & 4,07 & 3,86 & 3,69 & 3,56 & 3,45 & 3,35 \\
\hline 2000 & 3,07 & 3,68 & 3,61 & 3,44 & 3,27 & 3,14 & 3,04 & 2,95 & 2,88 \\
\hline 3000 & 2,67 & 3,32 & 3,28 & 3,13 & 2,99 & 2,88 & 2,78 & 2,71 & 2,64 \\
\hline 4000 & 2,44 & 3,09 & 3,07 & 2,94 & 2,81 & 2,71 & 2,62 & 2,55 & 2,49 \\
\hline 5000 & 2,27 & 2,94 & 2,93 & 2,80 & 2,68 & 2,59 & 2,51 & 2,44 & 2,39 \\
\hline 6000 & 2,15 & 2,82 & 2,82 & 2,70 & 2,59 & 2,49 & 2,42 & 2,36 & 2,31 \\
\hline 7000 & 2,05 & 2,72 & 2,73 & 2,62 & 2,51 & 2,42 & 2,35 & 2,29 & 2,24 \\
\hline 8000 & 1,97 & 2,64 & 2,65 & 2,55 & 2,44 & 2,36 & 2,29 & 2,23 & 2,19 \\
\hline 9000 & 1,90 & 2,58 & 2,59 & 2,49 & 2,39 & 2,31 & 2,24 & 2,19 & 2,14 \\
\hline 10000 & 1,84 & 2,52 & 2,53 & 2,44 & 2,34 & 2,26 & 2,20 & 2,14 & 2,10 \\
\hline 20000 & 1,52 & 2,19 & 2,22 & 2,14 & 2,06 & 1,99 & 1,94 & 1,90 & 1,86 \\
\hline 30000 & 1,37 & 2,02 & 2,06 & 1,99 & 1,92 & 1,86 & 1,81 & 1,77 & 1,74 \\
\hline 40000 & 1,27 & 1,92 & 1,96 & 1,89 & 1,82 & 1,77 & 1,73 & 1,69 & 1,66 \\
\hline 50000 & 1,20 & 1,84 & 1,88 & 1,82 & 1,76 & 1,71 & 1,66 & 1,63 & 1,60 \\
\hline 60000 & 1,15 & 1,78 & 1,82 & 1,77 & 1,71 & 1,66 & 1,62 & 1,58 & 1,55 \\
\hline 70000 & 1,11 & 1,74 & 1,78 & 1,72 & 1,66 & 1,62 & 1,58 & 1,55 & 1,52 \\
\hline 80000 & 1,07 & 1,70 & 1,74 & 1,69 & 1,63 & 1,58 & 1,55 & 1,51 & 1,49 \\
\hline 90000 & 1,04 & 1,66 & 1,71 & 1,65 & 1,60 & 1,55 & 1,52 & 1,49 & 1,46 \\
\hline 100000 & 1,02 & 1,63 & 1,68 & 1,63 & 1,57 & 1,53 & 1,49 & 1,46 & 1,44 \\
\hline 500000 & 0,72 & 1,27 & 1,31 & 1,28 & 1,24 & 1,21 & 1,19 & 1,17 & 1,15 \\
\hline 1000000 & 0,63 & 1,15 & 1,19 & 1,16 & 1,13 & 1,10 & 1,08 & 1,06 & 1,05 \\
\hline 5000000 & 0,47 & 0,93 & 0,97 & 0,95 & 0,92 & 0,90 & 0,89 & 0,87 & 0,86 \\
\hline 10000000 & 0,42 & 0,86 & 0,89 & 0,87 & 0,85 & 0,84 & 0,82 & 0,81 & 0,80 \\
\hline 50000000 & 0,32 & 0,72 & 0,75 & 0,73 & 0,72 & 0,70 & 0,69 & 0,68 & 0,67 \\
\hline 100000000 & 0,29 & 0,67 & 0,70 & 0,68 & 0,67 & 0,65 & 0,64 & 0,64 & 0,63 \\
\hline 500000000 & 0,24 & 0,57 & 0,59 & 0,58 & 0,57 & 0,56 & 0,55 & 0,55 & 0,54 \\
\hline 1000000000 & 0,22 & 0,53 & 0,56 & 0,55 & 0,53 & 0,53 & 0,52 & 0,51 & 0,51 \\
\hline
\end{tabular}

Le cas où $\alpha=99 \%$ ne sera donc pas traité. On obtient donc le tableau 1 de comparaison suivant.

Le tableau 1 précédent montre clairement que pour une valeur de risque de dépassement de $1 \%$, le modèle «asymptotique » permet de bien évaluer les valeurs du plus grand maxima local réduit d'un processus aléatoire gaussien stationnaire, pour autant que le nombre moyen de maxima locaux du p.a reste supérieur à 500 et ceci quelle que soit la largeur de bande du p.a (écart de l'ordre de $5 \%$ ).

\subsection{Cas d'un processus aléatoire à bande étroite}

En posant $r=1$ dans l'expression (67), on obtient l'expression asymptotique du plus grand maxima local réduit $\nu_{n, \alpha}$ pour le cas des processus aléatoires gaussiens à bande étroite, qui est un résultat déjà présenté dans [1]. On obtient donc l'expression suivante :

$$
\begin{aligned}
& \nu_{n, \alpha} \underset{n \text { grand }}{\longrightarrow} \sqrt{2 \ln (n)}-\frac{\ln [-\ln (1-\alpha)]}{\sqrt{2 \ln (n)}} \text { pour } r=1 \\
& \text { avec } n=E\left[\overline{N_{\max }}(\xi)\right] T=E\left[\overline{N_{0}^{+}}(\xi)\right] T
\end{aligned}
$$

\subsection{Cas d'un processus aléatoire à bande large}

La formulation (67) ne peut pas s'appliquer directement au cas des processus aléatoires à bande large $(r=0)$. Pour ce faire, il convient d'utiliser, pour $\nu_{n}$ et $\beta_{n}$, les 
expressions obtenues respectivement aux relations et (54). Ainsi on peut écrire :

$$
\begin{aligned}
\nu_{n, \alpha} \underset{n \text { grand }}{\longrightarrow} \nu_{n}-\frac{\ln [-\ln (1-\alpha)]}{\beta_{n}} \\
\text { et } \nu_{n} \approx \sqrt{-\frac{\pi}{2} \ln \left[\frac{4}{n}\left(1-\frac{1}{n}\right)\right]} \\
\beta_{n} \approx \frac{n}{\sqrt{2 \pi}} \exp \left[\frac{\pi}{4} \ln \left[\frac{4}{\pi}\left(1-\frac{1}{n}\right)\right]\right]
\end{aligned}
$$

\subsection{Valeur de risque de dépassement $\alpha_{0}$ associé à la définition du facteur de pic}

En comparant l'expression asymptotique de $\nu_{\alpha, n}$ définie par (67) à celle du facteur de pic $\delta_{z}$ définie par (62), il est possible de déterminer la valeur de risque de dépassement $\alpha_{0}$ qui est implicitement associée à la définition du facteur de pic. On a en effet :

$$
\varepsilon=-\ln \left[-\ln \left(1-\alpha_{0}\right)\right]
$$

avec $\varepsilon=$ Cste d'Euler $=0,57721566490 \ldots$

d'où la valeur de risque recherchée :

$$
\alpha_{0}=1-\exp [-\exp (-\varepsilon)] \approx 43 \% \forall r
$$

Le risque de dépassement associé à la définition du facteur de pic d'un p.a gaussien stationnaire étant de $43 \%$, quelle que soit sa largeur de bande, il convenait de s'appuyer favorablement sur la définition (67) du $\nu_{\alpha, n}$ plutôt que sur celle du facteur de pic $\delta_{z}$ pour sécuriser la démarche de conception des structures mécaniques, où l'on vise ici un risque voisin de $1 \%$ voire $0,1 \%$.

\section{Démarche de conception « sécurisante » retenue}

Sur la base de la théorie exposée précédemment, il est possible de mettre en place une démarche de conception sécurisante des structures mécaniques, en termes de contraintes extrêmes, avec pour optique de pouvoir dimensionner très rapidement et « sans risque » une structure mécanique soumise à plusieurs situations d'excitation aléatoires issues d'un profil d'emploi plus ou moins complexe. Dans le cas présent, on se restreint au cas des situations d'utilisation caractérisables par un processus aléatoire stationnaire de durée finie et de nature gaussienne, comme discuté dans les paragraphes précédents. De ces considérations techniques, chaque situation d'utilisation $S_{i}$ sera donc caractérisée par sa DSP d'accélération unilatérale $D S P_{\ddot{x} \ddot{x}}^{i}(f)$ de durée finie $T_{i}$.

Par ailleurs le comportement modal de la structure mécanique n'étant pas a priori connu au tout début du développement (le concept d'architecture de la structure à dimensionner étant non encore défini), la démarche de conception doit être capable de faire abstraction

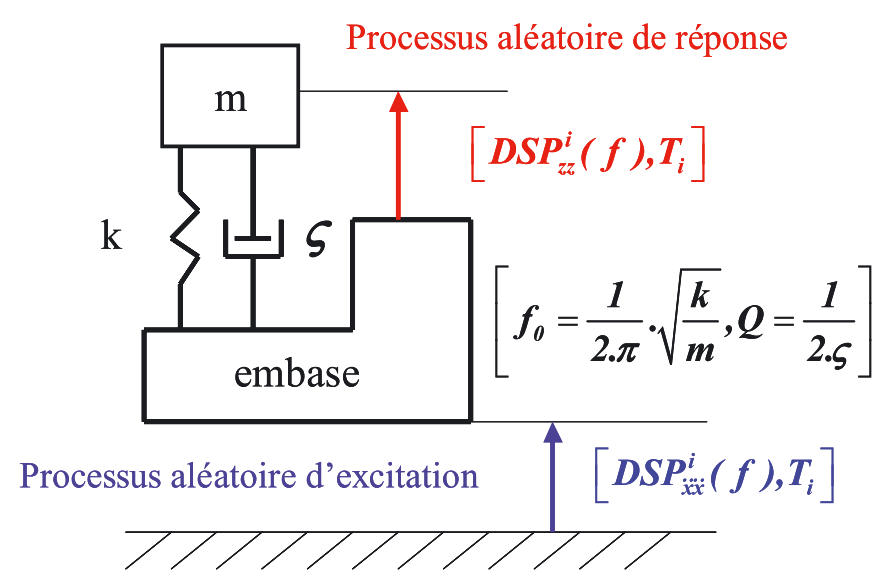

Fig. 14. Système étalon à 1 d.d.l.

du comportement dynamique réel de la structure, tout en permettant de caractériser l'ordre de grandeur des contraintes extrêmes induites par les situations d'excitation aléatoires $S_{i}$ considérées. Pour ce faire, on va donc modéliser le comportement dynamique de la structure par un système M.D.D.L (multi degré de liberté), comprenant un ensemble de modes découplés $f_{0}$ dans une bande de fréquence large, que l'on répartit entre une limite basse $f_{\mathrm{b}}$ et une limite haute $f_{\mathrm{h}}$, dépendant du support fréquentiel des $D S P_{\ddot{x} \ddot{x}}^{i}(f)$ considérées. De ces considérations techniques, il ressort donc que les contraintes extrêmes induites par les situations $S_{i}$ sur la structure mécanique à dimensionner seront évaluées au travers d'un comportement dynamique de structure simple comprenant un mode (assimilable à un système à 1 d.d.l), dont la fréquence propre $f_{0}$ sera déplacée dans la bande d'intérêt $\left[f_{\mathrm{b}}, f_{\mathrm{h}}\right]$. Cette philosophie est d'ailleurs celle qui est retenue dans le cas des méthodes de personnalisation, utilisées dans le contexte de la spécification des essais en environnement mécanique, pour la qualification des équipements embarqués [2].

\subsection{Système étalon à 1 d.d.I}

Compte tenu des considérations techniques précédentes, le processus aléatoire stationnaire gaussien à durée finie $\left[D S P_{\ddot{x} \ddot{x}}^{i}(f), T_{i}\right]$, caractérisant la situation d'excitation $S_{i}$, sera appliqué à l'embase du système étalon de fréquence propre $f_{0}$ et de coefficient de surtension $Q$ donnés, comme présenté à la figure 14 .

Pour caractériser le processus d'excitation aléatoire $\left[D S P_{\ddot{x} \ddot{x}}^{i}(f), T_{i}\right]$ de la situation d'utilisation $S_{i}$, en termes de contraintes extrêmes, on se propose de retenir le processus aléatoire de réponse $\left[D S P_{z z}^{i}(f), T_{i}\right]$ qui est associé au déplacement relatif du système étalon considéré, dans la mesure où ce dernier constitue l'image de la contrainte dynamique générée dans la structure, à un facteur multiplicatif $K$ près.

Ainsi connaissant le processus aléatoire d'excitation $\ddot{X}_{i}(t, \xi)$ défini par $\left[D S P_{\ddot{x} \ddot{x}^{i}}(f), T_{i}\right]$, il est possible de 
calculer au travers du système étalon $\left(f_{0}, Q\right)$ les caractéristiques du processus de réponse en contrainte $Z_{i}(t, \xi)$ en se basant sur la fonction de transfert du système étalon définie en terme de déplacement relatif/accélération absolue [14].

\subsection{Variable aléatoire retenue, au niveau du processus aléatoire de réponse}

Comme présenté précédemment et pour chaque situation $S_{i}$ considérée, le processus aléatoire de réponse $Z_{i}(t, \xi)$ sera caractérisé par sa variable aléatoire $Y_{n_{i}}^{i}(\xi)$, qui correspond au plus grand des $n_{i}$ maxima locaux du processus aléatoire de réponse $Z_{i}(t, \xi)$ considéré. On a donc :

$$
Y_{n_{i}}^{i}(\xi)=\underset{j=1}{\operatorname{MAX}_{i}}\left[Z_{\max , j}^{i}(\xi)\right]
$$

avec $\quad n_{i}=E\left[\overline{N_{\max }^{i}}(\xi)\right] T_{i}$

où :

$E\left[\overline{N_{\max }^{i}}(\xi)\right]$ : taux moyen des maxima locaux du processus aléatoire $Z_{i}(t, \xi)$,

$T_{i}$ : durée du processus de réponse $Z_{i}(t, \xi)$ et donc d'excitation $\ddot{X}_{i}(t, \xi)$

$Z_{\max , j}^{i}(\xi): j^{\text {ème }}$ maxima local du processus aléatoire $Z_{i}(t, \xi)$.

Sur la base de la théorie présentée précédemment, cette variable aléatoire $Y_{n_{i}}^{i}(\xi)$ est donc caractérisable sous forme probabiliste, en calculant sa fonction de répartition $F_{Y_{n_{i}}^{i}}(y)$ et/ou sa loi de densité de probabilité $f_{Y_{n_{i}}^{i}}(y)$. Pour ce faire, on se base respectivement sur les expressions générales (18) et (20) précédentes. Ce calcul est effectué pour chaque système étalon $\left(f_{0}, Q\right)$, dont la fréquence propre $f_{0}$ est comprise dans la bande d'intérêt $\left[f_{b}, f_{h}\right]$.

De ce fait pour chaque situation d'excitation $S_{i}$ donnée et chaque système étalon $\left(f_{0}, Q\right)$ considéré, le processus de réponse $Z_{i}(t, \xi)$ est caractérisé par la statistique des valeurs extrêmes, définie au travers de la variable aléatoire $Y_{n_{i}}^{i}(\xi)$ et de sa fonction de répartition $F_{Y_{n_{i}}^{i}}(y)$ :

$$
F_{Y_{n_{i}}^{i}}(y)=\left[F_{Z_{\max }^{i}}(y)\right]^{n_{i}} \quad \text { avec } \quad n_{i}=E\left[\overline{N_{\max }^{i}}(\xi)\right] T_{i}
$$

où :

$F_{Z_{\max }^{i}}(y)$ : Fonction de répartition des maxima locaux du processus aléatoire $Z_{i}(t, \xi)$, définie par l'expression (15).

\subsection{Variable aléatoire retenue, pour une situation d'utilisation associée à une valeur d'occurrence donnée}

Les profils d'emploi des clients sont en général décrits par un enchaînement de situations $S_{i}$, situées en série ou en parallèle, avec pour certaines d'entre elles des valeurs

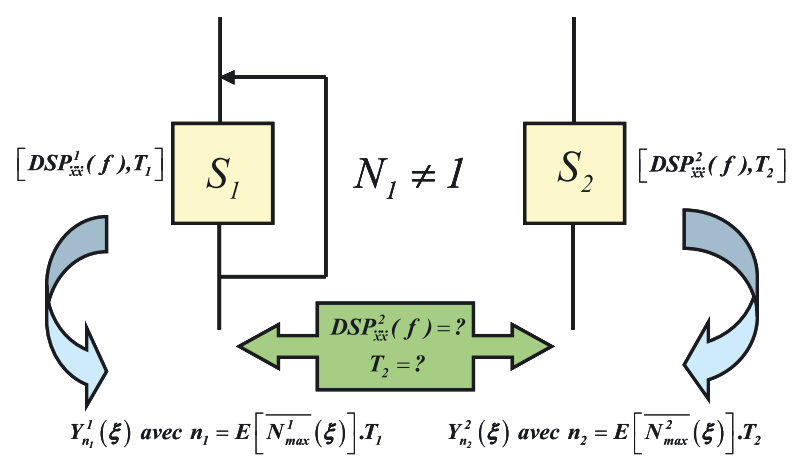

Fig. 15. Synthèse d'une situation d'utilisation associée à une occurrence non unitaire.

d'occurrence $\left(N_{i} \neq 1\right)$, qui correspondent aux valeurs de répétition des situations concernées.

Dans l'optique de synthétiser les profils d'emploi clients, décrits par un enchaînement parfois complexe de situations, en une seule situation d'excitation, il convient de savoir remplacer $N_{1}$ situations $S_{1}$ par une unique situation $S_{2}$, comme présenté à la figure 15 .

Ainsi en faisant l'hypothèse que les situations d'utilisation $S_{1}$ sont statistiquement indépendantes entre elles, il est possible d'écrire d'emblée :

$$
Y_{n_{2}}^{2}(\xi)=\underset{j=1}{\mathrm{NAX}_{1}}\left[Y_{n_{1}, j}^{1}(\xi)\right]
$$

avec $\quad n_{1}=E\left[\overline{N_{\max }^{1}}(\xi)\right] T_{1}$

où :

$E\left[\overline{N_{\max }^{1}}(\xi)\right]$ : taux moyen des maxima locaux du processus aléatoire $Z_{1}(t, \xi)$,

$T_{1}$ : durée du processus de réponse $Z_{1}(t, \xi)$ et donc d'excitation $\ddot{X}_{1}(t, \xi)$,

$n_{1}$ : nombre moyen de maxima locaux du processus de réponse $Z_{1}(t, \xi)$,

$Y_{n_{1}, j}^{1}(\xi): j^{\text {ème }}$ plus grand maxima local associé à la situation d'utilisation $S_{1}$,

$N_{1}$ : valeur d'occurrence associée à la situation $S_{1}$.

En s'appuyant sur l'expression (74), on peut écrire d'emblée que :

sachant que $\quad F_{Y_{n_{1}}^{1}}(y)=\left[F_{Z_{\max }^{1}}(y)\right]^{n_{1}}$

On obtient donc :

$$
F_{Y_{n_{2}}^{2}}(y)=\left[F_{Z_{\max }^{1}}(y)\right]^{n_{1} N_{1}}=\left[F_{Z_{\max }^{2}}(y)\right]^{n_{2}}
$$

sachant que $n_{2}=E\left[\overline{N_{\max }^{2}}(\xi)\right] T_{2}$

Ainsi pour satisfaire la relation (76) précédente, il faut que les deux conditions suivantes soient satisfaites, à savoir :

$$
\left\{\begin{aligned}
F_{Z_{\max }^{2}}(y) & =F_{Z_{\max }^{1}}(y) \\
n_{2} & =n_{1} N_{1}
\end{aligned}\right.
$$


Pour satisfaire l'égalité des fonctions de répartition, il suffit d'assurer l'égalité des DSP d'excitation entre la situation $S_{1}$ et la situation $S_{2}$. En effet, l'égalité des DSP d'excitation permet de travailler avec des moments spectraux (7) identiques au niveau des deux situations $S_{1}$ et $S_{2}$. Cela permet également de travailler avec des facteurs d'irrégularité identiques au niveau de ces deux situations, en s'appuyant sur (8).

De plus, l'égalité des DSP d'excitation conduit à satisfaire l'égalité des taux de maxima des deux situations $S_{1}$ et $S_{2}$, à savoir : $E\left[\overline{N_{\max }^{1}}(\xi)\right]=E\left[\overline{N_{\max }^{2}}(\xi)\right]$.

De ce fait les conditions (77) conduisent à écrire :

$$
\left\{\begin{aligned}
D S P_{\ddot{x} \ddot{x}}^{1}(f) & =D S P_{\ddot{x} \ddot{x}}^{2}(f) \\
T_{2} & =N_{1} T_{1}
\end{aligned}\right.
$$

À ce stade, il est possible de tirer les trois conclusions importantes suivantes, à savoir :

- Une situation $S_{1}$ de $D S P_{\ddot{x} \ddot{x}}^{1}(f)$, de durée $T_{1}$ et d'occurrence $N_{1} \neq 1$ peut être remplacée sur le plan statistique par une unique situation $S_{2}$ de même DSP $\left(D S P_{\ddot{x} \ddot{x}}^{2}(f)=D S P_{\ddot{x} \ddot{x}}^{1}(f)\right)$ et de durée $T_{2}=N_{1} T_{1}$.

- La statistique des maxima locaux est identique pour les deux situations $S_{1}$ et $S_{2}$, puisque l'on a : $F_{Z_{\max }^{2}}(y)=F_{Z_{\max }^{1}}(y)$.

- La statistique des plus grands maxima locaux est différente pour les deux situations $S_{1}$ et $S_{2}$, puisque l'on a : $F_{Y_{n_{2}}^{2}}(y)=\left[F_{Y_{n_{1}}^{1}}(y)\right]^{N_{1}}$.

\subsection{Variable aléatoire retenue, pour deux situations $S_{1}$ et $S_{2}$, d'occurrence unitaire et situées en série}

À ce stade, on recherche une situation $S_{3}$ telle que, sur le plan de la statistique des valeurs extrêmes, cette dernière soit équivalente aux deux situations $S_{1}$ et $S_{2}$, situées en série. Ces deux situations sont caractérisées en termes de contraintes extrêmes par respectivement les variables aléatoires $Y_{n_{1}}^{1}(\xi)$ et $Y_{n_{2}}^{2}(\xi)$, comme le montre la figure 16.

Dans l'optique de synthétiser les profils d'emploi clients, décrits par un enchaînement parfois complexe de situations, en une seule situation d'excitation, il convient de savoir remplacer deux situations $S_{1}$ et $S_{2}$, d'occurrence unitaire et situées en série, par une unique situation $S_{3}$, comme présenté à la figure 16 .

Ainsi en faisant l'hypothèse que les deux situations d'utilisation $S_{1}$ et $S_{2}$ sont statistiquement indépendantes, il est possible de déterminer la statistique des valeurs extrêmes de la situation $S_{3}$, connaissant celles des situations $S_{1}$ et $S_{2}$. En effet, on peut écrire :

$$
Y_{n_{3}}^{3}(\xi)=\operatorname{MAX}\left[Y_{n_{1}}^{1}(\xi), Y_{n_{2}}^{2}(\xi)\right]
$$

avec $n_{1}=E\left[\overline{N_{\max }^{1}}(\xi)\right] T_{1}$

et $n_{2}=E\left[\overline{N_{\max }^{2}}(\xi)\right] T_{2}$

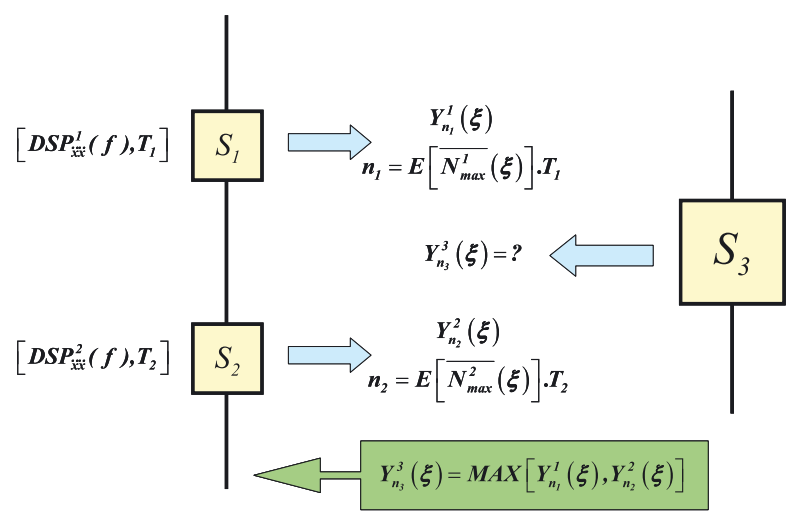

Fig. 16. Synthèse de deux situations d'utilisation situées en série.

où :

$E\left[\overline{N_{\max }^{1}}(\xi)\right]$ : taux moyen des maxima locaux du processus aléatoire $Z_{1}(t, \xi)$,

$T_{1}$ : durée du processus de réponse $Z_{1}(t, \xi)$ et donc d'excitation $\ddot{X}_{1}(t, \xi)$,

$n_{1}$ : nombre moyen de maxima locaux du processus de réponse $Z_{1}(t, \xi)$,

$E\left[\overline{N_{\max }^{2}}(\xi)\right]$ : taux moyen des maxima locaux du processus aléatoire $Z_{2}(t, \xi)$,

$T_{2}$ : durée du processus de réponse $Z_{2}(t, \xi)$ et donc d'excitation $\ddot{X}_{2}(t, \xi)$,

$n_{2}$ : nombre moyen de maxima locaux du processus de réponse $Z_{2}(t, \xi)$,

$Y_{n_{3}}^{3}(\xi)$ : plus grand maxima local associé à la situation d'utilisation $S_{3}$,

En s'appuyant sur l'expression (79), on peut donc écrire d'emblée que :

$$
\begin{aligned}
F_{Y_{n_{3}}^{3}}(y) & =\left[F_{Y_{n_{1}}^{1}}(y)\right]\left[F_{Y_{n_{2}}^{2}}(y)\right] \\
\text { avec } F_{Y_{n_{1}}^{1}}(y) & =\left[F_{Z_{\max }^{1}}(y)\right]^{n_{1}} \text { et } F_{Y_{n_{2}}^{2}}(y)=\left[F_{Z_{\max }^{2}}(y)\right]^{n_{2}}
\end{aligned}
$$

Ainsi la statistique des valeurs extrêmes de la situation $S_{3}$, qui est gouvernée par la variable aléatoire $Y_{n_{3}}^{3}(\xi)$, est calculable en terme de fonction de répartition, en utilisant l'expression (80) précédente. De ce fait il est possible, en s'appuyant sur les expressions (30) et (80), de calculer la valeur du plus grand maxima local $y_{n_{3}, \alpha}^{3}$, associée à la situation $S_{3}$ et dépassée avec un risque $\alpha$ donné. On peut écrire en effet :

$$
F_{Y_{n_{3}}^{3}}\left(y_{n_{3}, \alpha}^{3}\right)=\left[F_{Y_{n_{1}}^{1}}\left(y_{n_{3}, \alpha}^{3}\right)\right]\left[F_{Y_{n_{2}}^{2}}\left(y_{n_{3}, \alpha}^{3}\right)\right]=1-\alpha
$$

d'où

$$
y_{n_{3}, \alpha}^{3}=F_{Y_{n_{3}}^{3}}^{-1}(1-\alpha)
$$

À ce stade, il est important de noter que la statistique des valeurs extrêmes de la situation $S_{3}$, définie par $y_{n_{3}, \alpha}^{3}$, n'est pas accessible en faisant l'enveloppe des valeurs de 


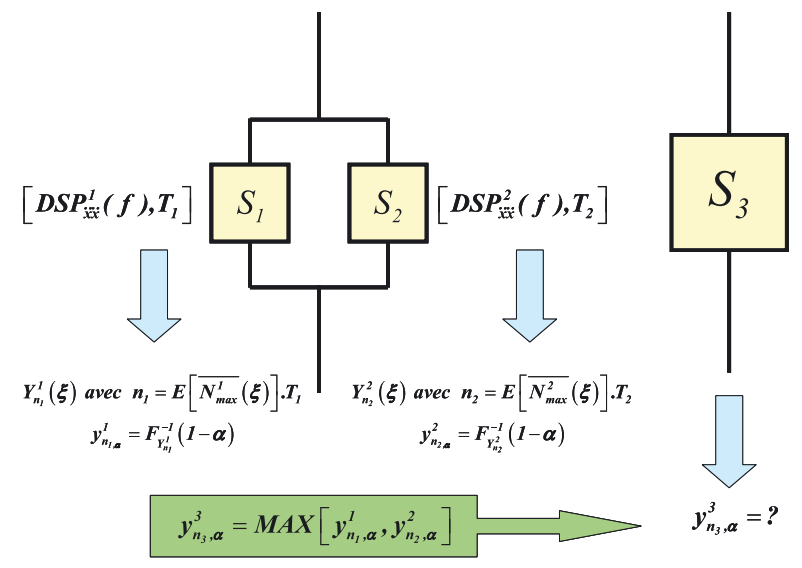

Fig. 17. Synthèse de deux situations d'utilisation situées en parallèle.

$y_{n_{1}, \alpha}^{1}$ et de $y_{n_{2}, \alpha}^{2}$, associées aux situations $S_{1}$ et $S_{2}$. Ainsi sur le plan statistique, il convient d'admettre que :

$$
y_{n_{3}, \alpha}^{3} \neq \operatorname{MAX}\left[y_{n_{1}, \alpha}^{1}, y_{n_{2}, \alpha}^{2}\right]
$$

avec $y_{n_{1}, \alpha}^{1}=F_{Y_{n_{1}}^{1}}^{-1}(1-\alpha)$ et $y_{n_{2}, \alpha}^{2}=F_{Y_{n_{2}}^{2}}^{-1}(1-\alpha)$

On montre aisément que l'expression (81), basée sur l'obtention de la f.d.r de la v.a $Y_{n_{3}}^{3}(\xi)$, conduit à évaluer une valeur de $y_{n_{3}, \alpha}^{3}$ majorante par rapport à la démarche consistant à réaliser l'enveloppe des valeurs $y_{n_{1}, \alpha}^{1}$ et $y_{n_{2}, \alpha}^{2}$, qui est couramment utilisée dans la pratique.

La généralisation de la formulation (81) pour $N$ situations $S_{i}$ statistiquement indépendantes, situées en série est :

$$
\begin{aligned}
F_{Y_{1}}\left(y_{1, \alpha}\right) & =\left[F_{Y_{n_{1}}^{1}}\left(y_{1, \alpha}\right)\right]\left[F_{Y_{n_{2}}^{2}}\left(y_{1, \alpha}\right)\right] \ldots\left[F_{Y_{n_{N}}^{N}}\left(y_{1, \alpha}\right)\right] \\
& =\prod_{i=1}^{N}\left[F_{Y_{n_{i}}^{i}}\left(y_{1, \alpha}\right)\right]=1-\alpha
\end{aligned}
$$

d'où $\quad y_{1, \alpha}=F_{Y_{1}}^{-1}(1-\alpha)$

où :

$Y_{1}(\xi)$ : plus grand maxima local, associé à l'ensemble des $N$ situations $S_{i}$ situées en série,

$y_{1, \alpha}$ : valeur du plus grand maxima local associé à la v.a $Y_{1}(\xi)$, qui est dépassée avec un risque $\alpha$.

\subsection{Variable aléatoire retenue, pour deux situations $S_{1}$ et $S_{2}$, d'occurrence unitaire et situées en parallèle}

À ce stade, on recherche une situation $S_{3}$ telle que, sur le plan de la statistique des valeurs extrêmes, cette dernière soit équivalente aux deux situations $S_{1}$ et $S_{2}$, situées en parallèle. Ces deux situations sont caractérisées en termes de contraintes extrêmes par respectivement les variables aléatoires $Y_{n_{1}}^{1}(\xi)$ et $Y_{n_{2}}^{2}(\xi)$, comme le montre la figure 17 qui suit. Si pour le cas de deux situations $S_{1}$ et $S_{2}$, situées en série, la définition d'une variable aléatoire
$Y_{n_{3}}^{3}(\xi)$ avait du sens sur le plan de la statistique des valeurs extrêmes, il n'en est pas de même pour le cas des situations en parallèle. Seule l'enveloppe des valeurs de $y_{n_{1}, \alpha}^{1}$ et de $y_{n_{2}, \alpha}^{2}$, associées aux situations $S_{1}$ et $S_{2}$ a du sens, dans le cas de deux situations en parallèle.

Ainsi sur le plan statistique, il convient d'écrire que :

$$
y_{n_{3}, \alpha}^{3}=\operatorname{MAX}\left[y_{n_{1}, \alpha}^{1}, y_{n_{2}, \alpha}^{2}\right]
$$

avec $y_{n_{1}, \alpha}^{1}=F_{Y_{n_{1}}^{1}}^{-1}(1-\alpha) \quad$ et $\quad y_{n_{2}, \alpha}^{2}=F_{Y_{n_{2}}^{2}}^{-1}(1-\alpha)$

La généralisation de la formulation (84) pour $N$ situations $S_{i}$ statistiquement indépendantes, situées en parallèle est :

$$
y_{1, \alpha}=\operatorname{MAX}\left[y_{n_{1}, \alpha}^{1}, y_{n_{2}, \alpha}^{2}, \ldots, y_{n_{N}, \alpha}^{N}\right]=\stackrel{\operatorname{MAX}_{i=1}^{N}}{N}\left[y_{n_{i}, \alpha}^{i}\right]
$$$$
\text { avec } \quad y_{n_{i}, \alpha}^{i}=F_{Y_{n_{i}}^{i}}^{-1}(1-\alpha)
$$

où :

$Y_{1}(\xi)$ : plus grand maxima local, associé à l'ensemble des N situations $S_{i}$ situées en parallèle,

$y_{1, \alpha}$ : valeur du plus grand maxima local associé à la v.a $Y_{1}(\xi)$, qui est dépassée avec un risque $\alpha$.

\subsection{Procédure d'établissement d'une spécification de conception « sécurisante »}

Ainsi, pour un profil d'emploi donné où chaque situation d'utilisation $S_{i}$ est définie par sa DSP et sa durée $\left[D S P_{\ddot{x} \ddot{x}}^{i}(f), T_{i}\right]$, on vient de montrer qu'il est alors possible d'en réaliser la synthèse au sens des valeurs extrêmes, en s'appuyant sur les méthodes développées aux sections 9.3-9.5. Ce processus de synthèse est réalisé pour chaque système étalon à 1 d.d.l de fréquence propre $f_{0}$ et de coefficient de surtension $Q$ donnés. De ce fait, la courbe de référence cible représentant la synthèse du profil d'emploi considéré, en termes de contraintes extrêmes, est fournie par le spectre de réponse extrême à risque de dépassement $\alpha$ [1] et [2], dénommé $S R X_{\text {cible }}\left(f_{0}\right)$, à savoir :

$$
S R X_{\text {cible }}\left(f_{0}\right)=\left(2 \pi f_{0}\right)^{2} y_{1, \alpha}\left(f_{0}\right)
$$

où :

$Y_{1}\left(f_{0}, \xi\right)$ : plus grand maxima local, associé à l'ensemble des $N$ situations $S_{i}$ du profil d'emploi considéré et caractérisé au travers du système étalon à 1 d.d.l $\left(f_{0}, Q\right)$,

$y_{1, \alpha}\left(f_{0}\right)$ : valeur du plus grand maxima local associé à la v.a $Y_{1}\left(f_{0}, \xi\right)$, qui est dépassée avec un risque $\alpha$ (homogène à un déplacement),

$S R X_{\text {cible }}\left(f_{0}\right)$ : spectre de réponse cible, associé aux valeurs de contraintes extrêmes générées par le profil d'emploi considéré, avec un risque de dépassement $\alpha$ donné.

Sachant que les processus de réponses, définis au travers des systèmes étalons à 1 d.d.l sont associés à des facteurs d'irrégularités proches de l'unité, il est possible de considérer que ces derniers sont essentiellement à bandes étroites. De ce fait, le processus de calcul des plus grands maxima locaux peut raisonnablement s'appuyer sur les 


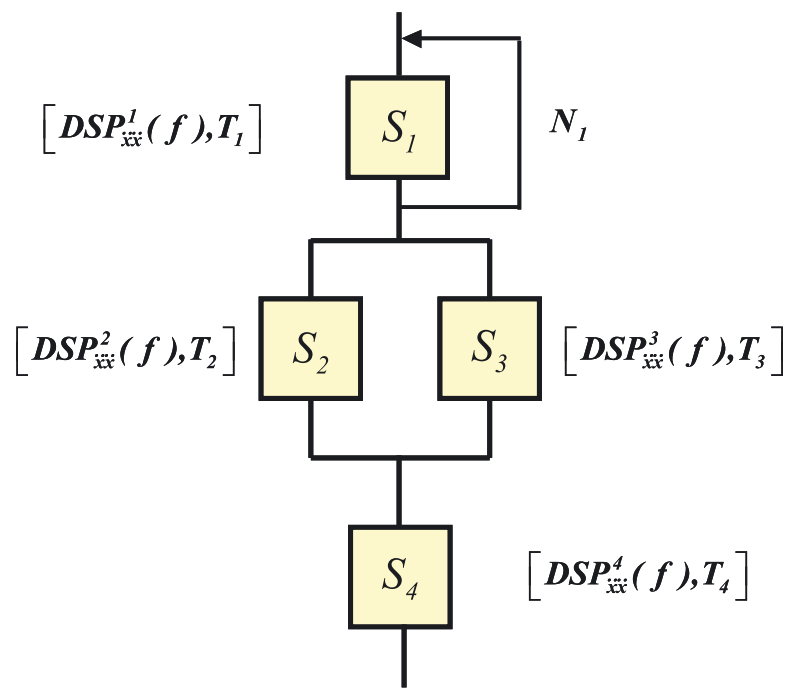

Fig. 18. Profil d'emploi opérationnel du cas d'application.

expressions asymptotiques (67) et (68) qui sont valides pour des valeurs de $\left(n=f_{0} T\right)$ proches de 200 dans le cas des processus à bandes étroites (voir Tab. 1).

De ces considérations techniques, il devient donc possible de calculer une spécification de conception, définie en terme de DSP par paliers $\left[D S P_{\ddot{x}_{c} \ddot{x}_{c}}^{1}(f), f \in\left[f_{b}, f_{h}\right]\right]$ pour une durée de sollicitation donnée $T_{c}$ et un risque $\alpha$ imposé, et dont son $S R X_{\text {conception }}\left(f_{0}\right)$ est identique au $S R X_{\text {cible }}\left(f_{0}\right)$ de référence. L'algorithme de « retour inverse » en terme de $S R X$ est développé autour des expressions asymptotiques (67) et (68), en utilisant un algorithme « itératif » dont le niveau de convergence s'avère à l'usage très rapide.

\section{Exemple d'application}

Pour illustrer les mécanismes de la démarche de conception exposée précédemment, on se propose de présenter ces derniers autour d'un cas d'application simple à traiter, auquel on associe des processus d'excitation fictifs, définis par des DSP de formes simples.

Cet exemple, bien que très simple, possède l'avantage d'illustrer les différents contours de la méthode en montrant les processus de synthèse des valeurs extrêmes sous toutes ses facettes, ainsi que le processus de retour inverse, défini en terme de $S R X$.

\subsection{Profil d'emploi opérationnel}

\subsection{Processus d'excitation, associés au profil d'emploi}

La forme des quatre DSP d'accélération est fournie sur la figure 20. On constate que l'enveloppe des DSP est fournie essentiellement par la DSP de la situation $S_{2}$ à basse fréquence et par la DSP de la situation $S_{4}$ à haute fréquence. De ce fait, il est clair que la DSP de conception sera logiquement gouvernée par ces deux DSP.

\begin{tabular}{|c|c|c|c|c|c|}
\hline \multirow{2}{*}{ Situation } & \multicolumn{2}{|c|}{ DSP d'excitation (constant par bande) } & \multirow{2}{*}{$\begin{array}{c}\text { Accèlération } \\
\text { efficace en }\left(m \cdot s^{2}\right)\end{array}$} & \multirow{2}{*}{ Occurrence } & \multirow{2}{*}{$\begin{array}{l}\text { Duree } \\
\text { (h) }\end{array}$} \\
\hline & frequence en $\mathrm{Hz}$ & niveau en $\left(\mathrm{m} \cdot \mathrm{s}^{2}\right)^{2} / \mathrm{Hz}$ & & & \\
\hline s1 & {$[5,500]$} & 0,01 & 2,22 & 10 & 1 \\
\hline \multirow{2}{*}{ \$2 } & {$[5,200]$} & 0,05 & \multirow{2}{*}{3,97} & \multirow{2}{*}{1} & \multirow{2}{*}{2} \\
\hline & {$[200,500]$} & 0,02 & & & \\
\hline \multirow{2}{*}{ s3 } & {$[5,100]$} & 0,005 & \multirow{2}{*}{2,91} & \multirow{2}{*}{1} & \multirow{2}{*}{10} \\
\hline & {$[100,500]$} & 0,02 & & & \\
\hline \multirow{3}{*}{$\$ 4$} & {$[5,300]$} & 0,02 & \multirow{3}{*}{3,45} & \multirow{3}{*}{1} & \multirow{3}{*}{3} \\
\hline & {$[300,400]$} & 0,04 & & & \\
\hline & {$[400,500]$} & 0,02 & & & \\
\hline
\end{tabular}

Fig. 19. Données d'excitation.

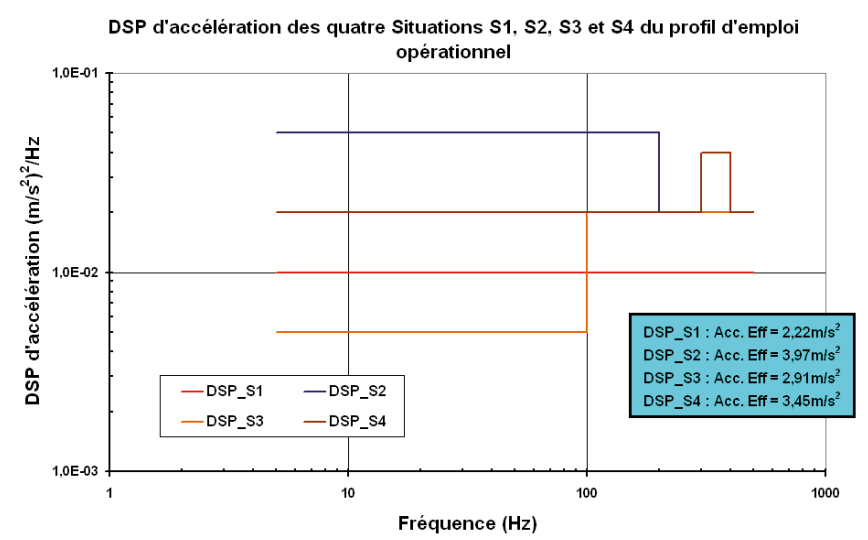

Fig. 20. DSP d'excitation (Figure disponible en couleurs sur ww. mecanique-industries.org).

\subsection{Processus de synthèse, associé au profil d'emploi}

Les deux situations $S_{2}$ et $S_{3}$ étant en parallèle, il convient donc de considérer que le profil d'emploi de la figure 18 peut se séparer en deux branches, comme présenté sur la figure 21.

De ce fait, on voit que la structure mécanique doit pouvoir être dimensionnée indifféremment aux cas des deux branches décrites à la figure 21 précédente. La branche B1 (branche de gauche sur la Fig. 21) est associée à la statistique des valeurs extrêmes décrite par la variable aléatoire $Y_{1}(\xi)$. Et la branche B2 (branche de droite sur la Fig. 21) est associée à la statistique des valeurs extrêmes décrite par la variable aléatoire $Y_{2}(\xi)$. Ces deux variables aléatoires $Y_{1}(\xi)$ et $Y_{2}(\xi)$ vont conduire à établir respectivement les deux spectres de réponses extrêmes à risque de dépassement $\alpha, S R X_{1}\left(f_{0}\right)$ et $S R X_{2}\left(f_{0}\right)$. Ainsi le processus d'obtention de ces deux spectres est décrit comme suit, à savoir :

$$
\left\{\begin{array}{l}
Y_{1}(\xi)=\operatorname{MAX}\left[Y_{n_{1}}^{1^{\prime}}(\xi), Y_{n_{2}}^{2}(\xi), Y_{n_{4}}^{4}(\xi)\right] \\
F_{Y_{1}}(y)=\left[F_{Y_{n_{1}}^{1^{\prime}}}(y)\right]\left[F_{Y_{n_{2}}^{2}}(y)\right]\left[F_{Y_{n_{4}}^{4}}(y)\right]
\end{array}\right.
$$




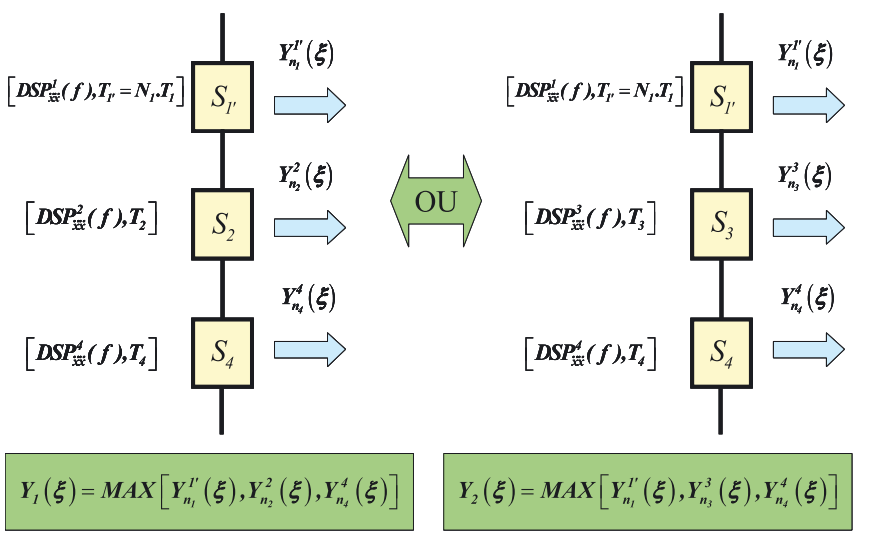

Fig. 21. Processus de synthèse, en termes de valeurs extrêmes.

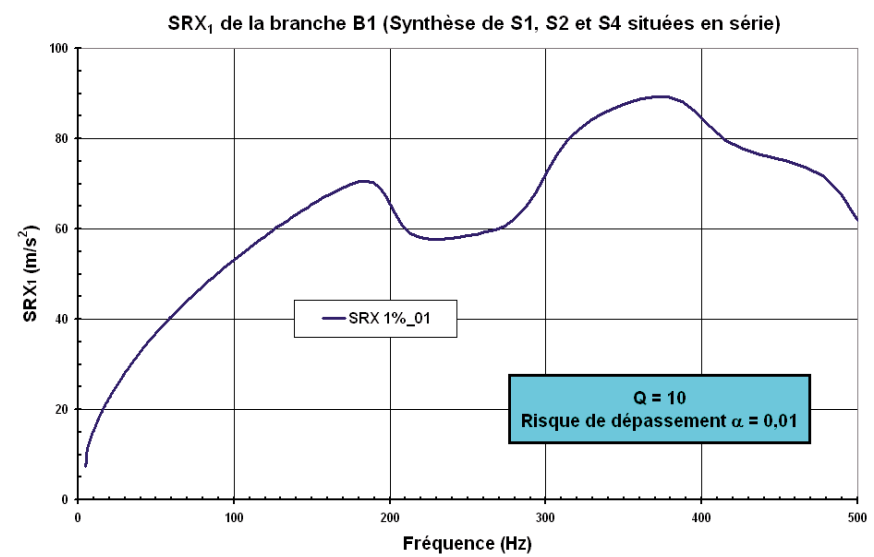

Fig. 22. $S R X_{1}\left(f_{0}\right)$ associé à la synthèse des situations de la branche B1.

avec $y_{1, \alpha}=F_{Y_{1}}^{-1}(1-\alpha)$ et $S R X_{1}\left(f_{0}\right)=\left(2 \pi f_{0}\right)^{2} y_{1, \alpha}\left(f_{0}\right)$ et,

$$
\left\{\begin{array}{l}
Y_{2}(\xi)=\operatorname{MAX}\left[Y_{n_{1}}^{1^{\prime}}(\xi), Y_{n_{3}}^{3}(\xi), Y_{n_{4}}^{4}(\xi)\right] \\
F_{Y_{1}}(y)=\left[F_{Y_{n_{1}}^{1^{\prime}}}(y)\right]\left[F_{Y_{n_{3}}^{3}}(y)\right]\left[F_{Y_{n_{4}}^{4}}(y)\right]
\end{array}\right.
$$

avec $y_{2, \alpha}=F_{Y_{2}}^{-1}(1-\alpha)$ et $S R X_{2}\left(f_{0}\right)=\left(2 \pi f_{0}\right)^{2} y_{2, \alpha}\left(f_{0}\right)$ La structure à dimensionner devant satisfaire au cas de la branche B1 ou au cas de la branche B2, le $S R X_{\text {cible }}\left(f_{0}\right)$ est alors obtenu en s'appuyant sur le processus de synthèse qui suit :

$$
y_{3, \alpha}=\operatorname{MAX}\left[y_{1, \alpha} ; y_{2, \alpha}\right]
$$

D'où : $y_{3, \alpha}\left(f_{0}\right)=\operatorname{MAX}\left[y_{1, \alpha}\left(f_{0}\right) ; y_{2, \alpha}\left(f_{0}\right)\right]$

et

$$
\begin{aligned}
& \operatorname{SRX}_{3}\left(f_{0}\right)=\underset{f_{0}=f_{b}}{\mathrm{fAX}_{h}}\left[S R X_{1}\left(f_{0}\right) ;\right. \\
& \left.S R X_{2}\left(f_{0}\right)\right]=S R X_{\text {cible }}\left(f_{0}\right)
\end{aligned}
$$

On obtient donc la figure 24 .

À ce stade, on constate que c'est la branche B1 qui est la plus critique en termes de valeurs extrêmes pour la

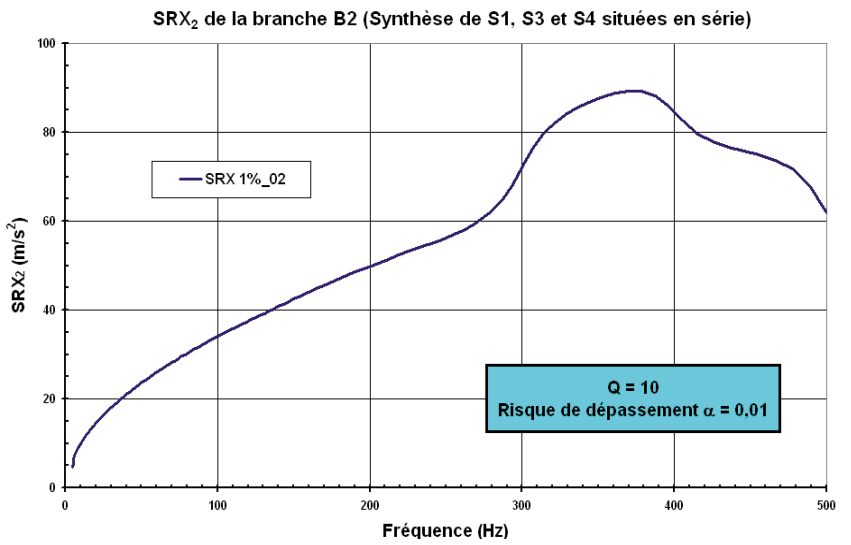

Fig. 23. $S R X_{2}\left(f_{0}\right)$ associé à la synthèse des situations de la branche B2.

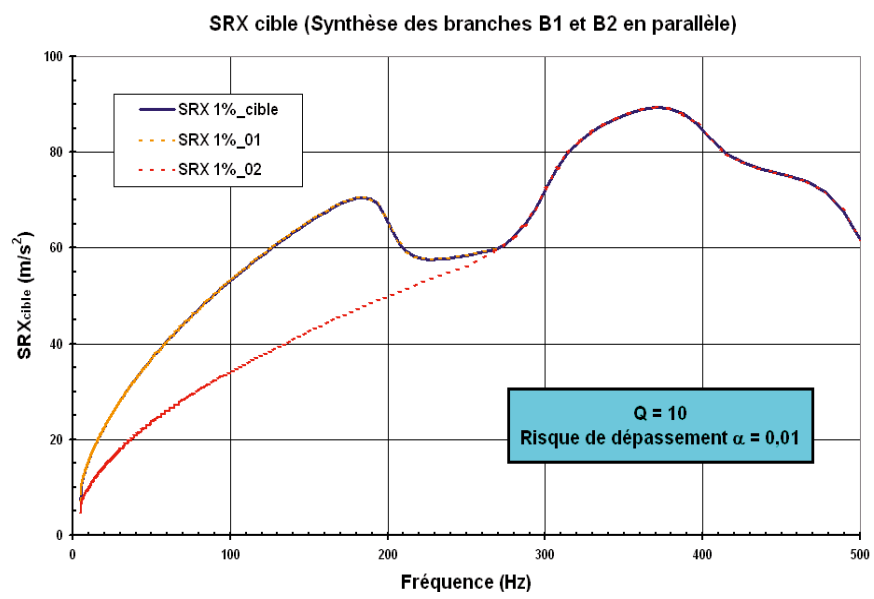

Fig. 24. $S R X_{\text {cible }}\left(f_{0}\right)$ associé à la synthèse des branches B1 et B2 du profil d'emploi opérationnel (Figure disponible en couleurs sur www.mecanique-industries.org).

conception de la structure mécanique considérée, et ceci quel que soit son comportement modal. Cette méthode de conception permet donc d'identifier les branches d'un profil d'emploi opérationnel qui s'avère les plus dimensionnantes pour une structure mécanique quelconque. De ces considérations techniques, cette méthode offre l'avantage, au tout début d'un programme de développement, d'affiner avec le client les données d'excitation (niveau et durée) qui s'avèrent les plus pertinentes pour le développement en cours.

\subsection{Processus de « retour inverse $»$ en terme de SRX}

Comme présenté en introduction, la démarche de conception « sécurisante » consiste à pouvoir synthétiser très vite un grand nombre de processus d'excitation aléatoire, au sens des valeurs de contraintes extrêmes à risque de dépassement $\alpha$ imposé, mais surtout de pouvoir revenir à une spécification de conception de nature 


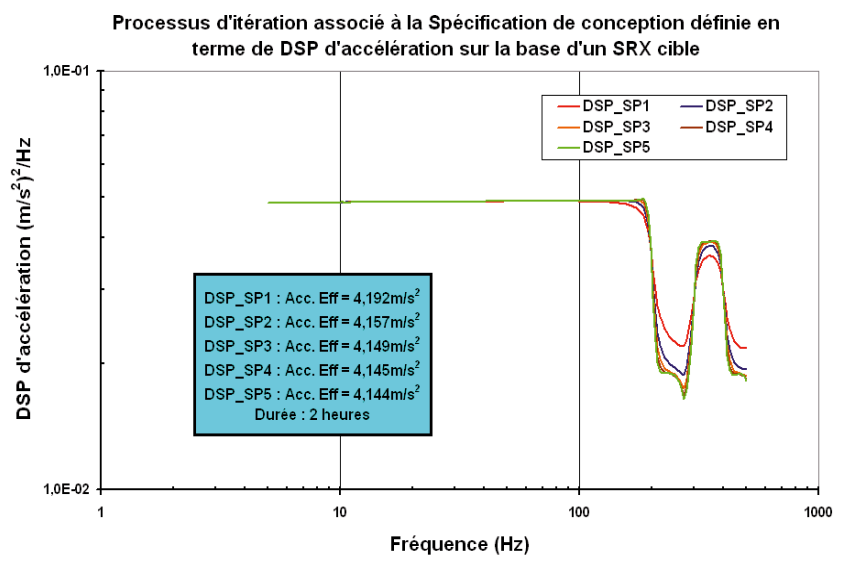

Fig. 25. Spécification de conception (duré 2 heures) (Figure disponible en couleurs sur www.mecanique-industries.org).

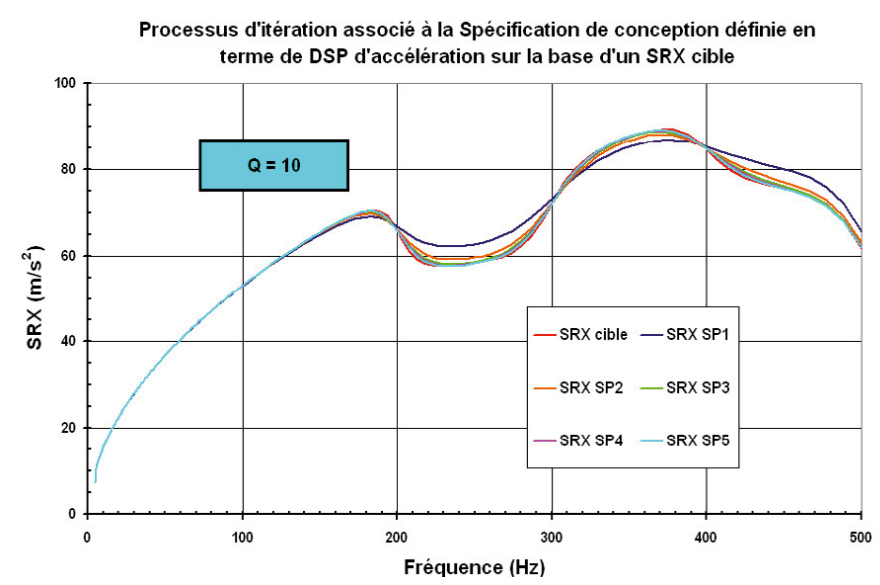

Fig. 26. Processus de convergence en SRX (Figure disponible en couleurs sur www.mecanique-industries.org).

dynamique et non plus statique (comme dans le passé), couvrant la synthèse des valeurs extrêmes du profil d'emploi opérationnel considéré. Pour ce faire, on s'appuie sur la procédure définie à la section 9.6 qui a été mise en place dans un outil «propriétaire » dédié à l'établissement de spécifications de conception « au juste nécessaire ». Pour le cas de notre exemple, le processus de « retour inverse» en terme de SRX, conduit au résultat suivant pour une durée de sollicitation cible, basée sur la durée opérationnelle la plus petite ( 2 heures), associée aux situations du profil d'emploi opérationnel.

En terme de SRX, le processus d'itération est présenté à la figure 26 suivante, montrant la vitesse de convergence importante du processus de « retour inverse » puisqu'en 5 itérations successives, on constate que l'on couvre avec une très bonne précision le SRX cible, issu de la synthèse.

Pour conclure sur le niveau de crédibilité de la démarche de conception présentée, on montre clairement, à partir de la figure 25, que le niveau efficace de la DSP de conception situé à $4,14 \mathrm{~m} . \mathrm{s}^{-2}$ pour $2 \mathrm{~h}$ de sollicitation, est une valeur cohérente au vu des valeurs efficaces associées aux quatre situations du profil d'emploi opérationnel (voir Fig. 19). Par ailleurs, on montre que l'allure de la DSP

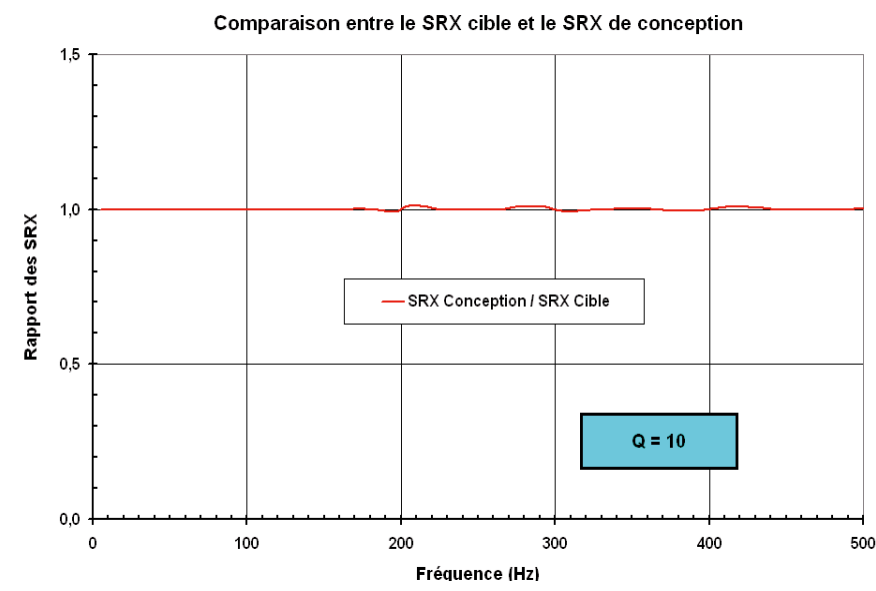

Fig. 27. Convergence du processus de « retour inverse $»$ en SRX (Figure disponible en couleurs sur www. mecanique-industries.org).

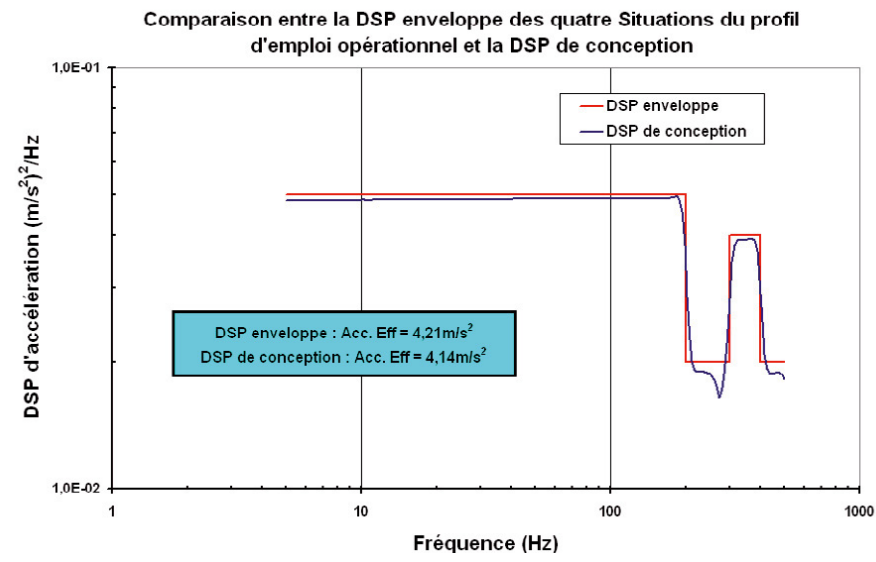

Fig. 28. Comparaison entre la DSP enveloppe et la DSP de conception (Figure disponible en couleurs sur ww. mecanique-industries.org).

de conception est cohérente avec celles des DSP d'excitation des quatre situations du profil d'emploi, puisque cette dernière est complètement calée sur l'enveloppe de ces quatre DSP d'excitation, comme le montre la figure 28.

\section{Références}

[1] B. Colin, La probabilisation des SRE : une réponse à la comparaison des sévérités d'environnement déterministe et stochastique, en termes de contraintes extrêmes, Septembre 2007, ASTELAB 2007, pp. 25-27

[2] DGA, Guide d'application de la démarche de personnalisation en environnement mécanique, Contrat de Normalisation NORM/06, Mars 2006

[3] Giat industries, Le VBCI sur les pistes, Giat Magazine $\mathrm{N}^{\circ} 55$, février 2004

[4] Giat industries, VBCI, a major programme, Giat Magazine $\mathrm{N}^{\circ}$ 56, Eurosatory 2004, June 2004

[5] B. Colin, Comportement non linéaire des suspensions mécaniques et oléopneumatiques montées sur véhicules blindés, ASTELAB 2001, 13 et 14 Mars 2001 
[6] S.O. Rice, Mathematical analysis of random noise, Bell System Techn. J. 28 (1944-1945)

[7] D.E Cartwright, M.S Longuet-Higgins, The distribution of the maxima of a random function, Proceedings of the Royal Society 237, 1956, pp. 212-232

[8] S. Colombo, Fonctions spéciales, A 152, Les Techniques de l'Ingénieur

[9] J.S. Bendat, A.G Piersol, Random Data, WileyInterscience, $2^{\mathrm{e}}$ édition, 1986

[10] G. Saporta, Probabilités, Analyse des données et statistiques, Editions. Technip, 1990, 495 p.
[11] A.B. De Vriendt, La transmission de la chaleur, in : G. Morin (éd.), Vol. 1, Tome 2, 1962

[12] E.J. Gumbel, Statistics of Extremes, Columbia University Press, 1958

[13] R.A. Fishe, L.H.C. Tippet, Limiting forms of the frequency distribution of the largest or smallest member of a sample, Poc. Camb. Phil. Soc. 24 (1928) 180

[14] S.H. Crandall, W.D. Mark, Random vibration in mechanical systems, Academic Press, 1963 